\title{
Bimodal behavior of the Kuroshio and the Gulf Stream
}

\author{
Maurice J. Schmeits and Henk A. Dijkstra \\ Institute for Marine and Atmospheric research Utrecht \\ Utrecht University \\ Utrecht, the Netherlands
}

Final version for Journal of Physical Oceanography

Corresponding author address:

Maurice Schmeits

Institute for Marine and Atmospheric research Utrecht

Department of Physics and Astronomy

Utrecht University

Princetonplein 5, 3584 CC Utrecht

The Netherlands

Phone: -31-30-2532265; Fax: -31-30-2543163

Email: schmeits@phys.uu.nl 


\begin{abstract}
For a long time, observations have been pointing out that the Kuroshio in the North Pacific Ocean displays bimodal meandering behavior off the southern coast of Japan. For the Gulf Stream in the North Atlantic Ocean, weakly and strongly deflected paths near the coast of South Carolina have been observed. This suggests that bimodal behavior may occur in the Gulf Stream as well, although less pronounced than in the Kuroshio. Evidence from a high resolution ocean general circulation model (OGCM) and intermediate complexity models is given to support the hypothesis that multiple mean paths of both the Kuroshio and the Gulf Stream are dynamically possible. These paths are found as multiple steady states in an intermediate complexity shallow-water model. In the OGCM, transitions between similar mean paths are found, with the patterns having similarity to the ones in the observations as well. To study whether atmospheric noise can induce transitions between the multiple steady states, a stochastic component is added to the annual mean wind stress forcing in the intermediate complexity model and differences between the transition behavior in the Gulf Stream and Kuroshio are considered.
\end{abstract}




\section{Introduction}

The Kuroshio in the North Pacific Ocean and the Gulf Stream in the North Atlantic Ocean are the major northern hemispheric western boundary currents which transport an enormous amount of volume and heat/salt polewards. The mean position of these currents is therefore important for the global climate system. Although the North Pacific basin has larger dimensions than the North Atlantic basin, the mean wind stress forcing is very similar and a close dynamical correspondence between both western boundary currents is expected.

From observations, it is found that the Kuroshio path exhibits bimodal behavior to the south of Japan with transitions occurring between a small and a large meander state (Fig. 1; adapted from Taft, 1972). Both states can persist over a period ranging from a few years to a decade and transitions between them occur within a couple of months (Kawabe, 1986). A 42-year long time series of the mean distance of the Kuroshio axis from the Japanese coast (Qiu and Miao, 2000) shows that large meander states occurred, for example, during 1959-1962, 1975-1979 and 1982-1985.

Satellite images of sea surface temperature (SST) of the North Atlantic have revealed that the Gulf Stream near South Carolina can be in a weakly deflected or a strongly deflected state (Fig. 2; from Bane and Dewar, 1988). Bane and Dewar (1988) have presented observations which suggest that the seaward deflection of the Gulf Stream has a bimodal character and that the transitions between both states occur on intermonthly timescales. Results from other studies of the Gulf Stream path using in situ measurements and very high resolution infrared satellite data also show indications of bimodal behavior. In Fig. 4 of Olson et al. (1983), a histogram of the cross-stream frontal position (over the period 1976-1980) shows a bimodal distribution in the area just before separation (at about $77^{\circ} \mathrm{W}$ ). Similarly, in Fig. 8c of Auer (1987) using AVHRR data over the period 1980-1985, a probability distribution of the Gulf Stream Landward Surface Edge shows an indication for bimodality in the area just after separation (at $71^{\circ} \mathrm{W}$ ). The two peaks in the histograms of Olson et al. (1983) and Auer (1987) are separated from each other by about $50 \mathrm{~km}$ in cross-stream direction and $0.5^{\circ}$ in latitude, respectively.

The question arising from these observations is clear: what are the physical processes responsible for this bimodal behavior? A central element in the classical explanation of the bimodality of the Kuroshio is the existence of multiple equilibria (Charney and Flierl, 1981; Masuda, 1982). In most of the previous modeling studies (e.g. Chao, 1984), regional models were used with in/outflow boundary conditions and multiple paths of the Kuroshio were found. For instance, using a barotropic quasigeostrophic model with geometry and bottom topographic features incorporated, Chao (1984) found multiple quasi-steady (small and large meander) paths of the Kuroshio if the volume transport exceeds a certain critical value. For values smaller than this critical transport, about 30 $\mathrm{Sv}$ in his model, only a small meander state is found. When the continental geometry representing the Kyushu wedge is removed, the large meander state can no longer be found, indicating that the geometry is crucial for the existence of the bimodality. For 
the Gulf Stream, Bane and Dewar (1988), following Legeckis (1976), have attributed the deflection to the flow of the Stream over the "Charleston bump", a topographic feature located on the upper continental slope at about $31^{\circ} \mathrm{N}$ offshore of Charleston, South Carolina.

Although not really challenging the multiple equilibria viewpoint of the bimodality of the Kuroshio, Qiu and Miao (2000) have pointed out that the path flow variations are not necessarily controlled by the external inflow changes. In fact, they have shown, using the relatively long (from 1960) in-situ observational time series, that there is no close correspondence between upstream volume transport and transitions in the mean path of the Kuroshio. Moreover, as Qiu and Miao (2000) have pointed out, regional models with in/outflow boundary conditions (e.g. Chao, 1984) may strongly influence the dynamics of the flow and not be able to realistically capture the Kuroshio's recirculation gyre, which is an inseparable part of the Kuroshio current system.

To study Kuroshio path transitions, Qiu and Miao (2000) have therefore chosen to use a 2-layer primitive-equation model of the North Pacific Ocean with a horizontal resolution of $\frac{1}{6}^{\circ}$ in a realistic configuration driven by monthly climatological wind stress data (Hellerman and Rosenstein, 1983). Variations between a straight path and a meander path of the Kuroshio are found on interannual timescales, when the wind forcing is strong enough. They propose that the observed alternations of the Kuroshio's two states are due to a self-sustained internal oscillation involving the evolution of the southern recirculation gyre and the stability of the Kuroshio current system, rather than being controlled by the temporal changes in the upstream Kuroshio transport. In one phase of the oscillation, low potential vorticity water of southern origin is advected with the inertially controlled Kuroshio path, which strengthens the southern recirculation gyre. This leads initially to the straight path, but as the intensification of the recirculation gyre progresses, it eventually leads to the development of a meander path due to baroclinic instability. As the meander grows in amplitude, it eventually breaks off to form an isolated eddy, thereby mixing high potential vorticity water of coastal origin into the recirculation gyre which weakens the latter. The Kuroshio will return to the straight path state after re-strengthening of the recirculation gyre due to the gradual accumulation of the low potential vorticity anomalies.

Hurlburt et al. (1996) have also used a primitive-equation model of the North Pacific in a realistic configuration, driven by the Hellerman and Rosenstein (1983) monthly wind stress climatology, but with 6 vertical layers and a horizontal resolution of $\frac{1}{8}^{\circ}$. Various transitions between a straight Kuroshio path and a meander Kuroshio path have been found, with each state lasting for several years; the model results show persistent bistable states with mesoscale variability but little variation in 1-year means. The major differences between the Hurlburt et al. (1996) results (their Figs. 6e and g) and those of Qiu and Miao (2000) (their Fig. 8) are that in the former results (i) the straight path is more zonally oriented, contrary to observations (cf. Fig. 1), and (ii) the zonal penetration scale of the Kuroshio Extension is much longer, probably as a result of the higher resolution. From the set of model runs in Hurlburt et al. (1996), they conclude that the meander path depends on the occurrence of baroclinic instability west 
of the Izu Ridge and that otherwise a straight path occurs. Increases in wind forcing on interannual timescales give rise to a predominant meander path, while decreases yield a predominant straight path.

Indications of bimodal behavior are also found in very high resolution simulations of the Gulf Stream (Smith et al., 2000), although it has not been recognized as such. For instance, during the spinup phase of a simulation using the Los Alamos Parallel Ocean Program (POP) model (with a horizontal resolution of $\frac{1}{10}^{\circ}$ and 40 vertical levels), the simulated Gulf Stream south of Cape Hatteras 'occasionally meandered too far from the coastline'. Because it was suggested that this might be caused by the inadequate resolution of the Munk layer, the viscosity and diffusivity were both increased by a factor of 3 to reduce this "problem" (Smith et al., 2000).

Much work has been done to understand the separation of the Gulf Stream near Cape Hatteras (Dengg et al., 1996; Özgökmen et al., 1997). In a high resolution Modular Ocean Model simulation with $\frac{1}{3}^{\circ}$ horizontal resolution (Beckmann et al., 1994; Bryan et al., 1995), the time mean state shows a large anti-cyclonic gyre north of Cape Hatteras giving an actual separation north of the observed position with non-desirable consequences for the simulated heat transport. However, the POP model shows realistic separation near Cape Hatteras (Smith et al., 2000, their Fig. 6). The mean position of the Gulf Stream, as defined by the $12^{\circ} \mathrm{C}$ isotherm at $400 \mathrm{~m}$ depth averaged over a four year period, shows only a slight southward offset of the path after separation. Also the Gulf Stream path statistics are reasonable, with the magnitude of the simulated meander envelope being similar to that of the observed envelope. Although many parameter studies which have been performed with idealized models cannot easily be done with the high resolution models, it is clear that when the resolution increases in the models and friction coefficients decrease, the inertial character of the flow increases and separation improves (McWillams, 1996; Hurlburt and Hogan, 2000).

In section 2 below, the behavior of the northern hemispheric western boundary currents is investigated in output from the Parallel Ocean Climate Model (POCM). Bimodality is found in both the Kuroshio and the Gulf Stream region although of different intensity and frequency. To find a theoretical framework to understand the bimodality in this model, we continue a line of studies addressing the stability and variability of western boundary currents within a hierarchy of models using techniques of dynamical systems theory (Speich et al., 1995; Dijkstra and Katsman, 1997; Dijkstra and Molemaker, 1999; Dijkstra et al., 1999; Schmeits and Dijkstra, 2000). Over the last decade, theoretical studies of behavior of nonlinear western boundary currents have indicated that multiple steady states of the wind-driven gyres appear in idealized closed rectangular basins through spontaneous symmetry breaking (Cessi and Ierley, 1995; Jiang et al., 1995; Dijkstra and Katsman, 1997). In (equivalent) barotropic models, asymmetric states are found under symmetric (with respect to the mid-basin axis) forcing conditions. These multiple steady states persist when continental geometry and realistic wind stress forcing are considered (Dijkstra and Molemaker, 1999). Given this robustness and the dynamical correspondence of Kuroshio and Gulf Stream, it is expected that in closed basin models for the North Pacific and North Atlantic, these 
multiple equilibria are present. Indeed, in section 4 below it is shown that qualitatively the bifurcation diagrams for both cases are the same within a barotropic shallow-water (SW) model (section 3), although the associated flow patterns and stability characteristics differ markedly. Moreover, the multiple paths have resemblance to those found in observations and in POCM.

Next, the possibility of transitions between the steady states through atmospheric noise is investigated. Sura et al. (2001) have used a reduced-gravity double-gyre ocean model for a rectangular basin to study the influence of an additive stochastic wind stress component on the behavior of the wind-driven circulation. When forced with a spatially inhomogeneous stochastic forcing, in order to mimic the observed concentration of atmospheric eddy activity along the storm track, the model shows bimodal behavior. In one regime a quasi-antisymmetric flow pattern is found, while the other regime shows a non-symmetric flow pattern. Although no stationary solutions have explicitly been calculated in their study, Sura et al. (2001) have suggested that the non-symmetric regime corresponds to one member of a known non-symmetric pair of stationary solutions. This is confirmed by comparing their snapshots of the non-symmetric regime to the "jet up" stationary solution of Speich et al. (1995), who have used a very similar model. Sura et al. (2001) have not found signatures of the symmetry related solution, that is the "jet down" stationary solution of Speich et al. (1995). Their study has indicated that the bimodality does not appear without stochastic forcing nor with spatially homogeneous stochastic forcing. They have therefore concluded that the regime transitions are induced by the inhomogeneity of the white noise variance. In section 5, we will investigate the effect of an additive stochastic wind stress component on the behavior of the North Atlantic and North Pacific wind-driven circulation in a more realistic model context than that of Sura et al. (2001).

\section{Northern hemispheric western boundary currents in POCM}

The basic POCM formulation has been described by Semtner and Chervin (1992) and Stammer et al. (1996). The POCM output analysed is from run 4C having an average horizontal resolution of $\frac{1}{4}^{\circ}$ and 20 nonequidistant levels in the vertical direction. The model contains bottom topography, extrapolated from a bathymetric dataset. The global simulation was performed over the period 1979-98 and the ocean was forced by either ECMWF reanalysis (1979-93) or ECMWF operational (1994-98) fields of heat fluxes, freshwater fluxes, and wind stress. These fields are updated every 3 days and interpolated to the time step. The annual river outflow was also included in the freshwater flux. Diffusion is handled using biharmonic closure for both momentum and tracers (Stammer et al., 1996). For more details about this simulation the reader is referred to http : //vislab - www.nps.navy.mil/ rtt. We have used monthly mean sea surface temperature (SST), sea surface height (SSH), and temperature fields at 
two depth levels, namely $310 \mathrm{~m}$ (T310) and $610 \mathrm{~m}$ (T610), in the Gulf Stream region $\left(90^{\circ}-50^{\circ} \mathrm{W}, 23^{\circ}-48^{\circ} \mathrm{N}\right)$ and in the Kuroshio region $\left(120^{\circ}-160^{\circ} \mathrm{E}, 25^{\circ}-50^{\circ} \mathrm{N}\right)$. $\mathrm{SSH}$ is a prognostic variable in POCM because of the incorporation of a free surface formulation.

\section{a. Bimodality of the Kuroshio}

In Fig. 3a, the latitudinal position of the maximum zonal geostrophic velocity at $136^{\circ} \mathrm{E}$ is plotted over the period 1979-1998, and several transitions between two states occur on interannual timescales. In Figs. 3b and c, SSH deviations are superimposed on SST for the two states. One state, called Kuroshio meander state $K_{b}^{P}$ (Fig. $3 \mathrm{~b}$ ), resembles the observed small meander state (Fig. 1). It is characterized by a northerly position of the maximum zonal geostrophic velocity at a latitude of about $33^{\circ} \mathrm{N}$ (Fig. 3a), accompanied by an anti-cyclonic recirculation cell to the south. The other state, denoted as Kuroshio meander state $K_{c}^{P}$ (Fig. 3c), resembles the observed large meander state (Fig. 1). It is characterized by a southerly position of the maximum zonal geostrophic velocity at a latitude of about $31.5^{\circ} \mathrm{N}$ (Fig. 3a), accompanied by a cyclonic recirculation cell to the north. In both cases the main separation latitude of the Kuroshio is too far north (at about $40^{\circ} \mathrm{N}$ ), as is also a problem in other high resolution models (e.g. Maltrud et al., 1998). By comparing the patterns of both states to the observed ones (cf. Fig. 1), the main differences are that the simulated small meander of $K_{b}^{P}$ has a larger amplitude than the observed one, and that the simulated large meander of $K_{c}^{P}$ has a smaller zonal extension than the observed one. Shaded contour plots of SST (Figs. 3b and c) indicate that heat is strongly advected with the mean current. The Kuroshio advects more heat northward in the case of small meander state $K_{b}^{P}$ (Fig. $3 \mathrm{~b}$ ) than in the case of large meander state $K_{c}^{P}$ (Fig. 3c).

The simulated Kuroshio has a larger eastward geostrophic transport when it is in its small meander state $\left(K_{b}^{P}\right)$ than when it is in its large meander state $\left(K_{c}^{P}\right)$, at least at $136^{\circ} \mathrm{E}$ (Fig. 3a). Observations point at geostrophic transports of equal magnitude for both states at $137^{\circ} \mathrm{E}$ (Qiu and Joyce, 1992). Finally, Fig. 3a suggests that the timing of the transitions from the large $\left(K_{c}^{P}\right)$ to the small $\left(K_{b}^{P}\right)$ meander state is related to the occurrence of strong El Niño events (beginning of 1983 and 1998). In other words, anomalous conditions during a strong El Niño (for instance in the pattern or amplitude of the wind stress field) may induce transitions from the large to the small meander state. Note, however, that the transitions between the two states occur at different times in POCM than those in reality (Kawabe, 1995).

In Fig. 4 a sequence of POCM temperature fields at depth during 1998 shows the evolution of the Kuroshio from the large $\left(K_{c}^{P}\right)$ to the small $\left(K_{b}^{P}\right)$ meander state and back (see Fig. 3a). The upper left panel shows the Kuroshio in its large meander state. The meander grows in amplitude in the subsequent months (upper right panel) and it eventually breaks off to form an isolated eddy (situated at about $\left(133^{\circ} \mathrm{E}, 28^{\circ} \mathrm{N}\right.$ ) in middle left panel), which drifts westward. After the detachment of the eddy, the Kuroshio path turns to the small meander state for a few months (middle panels). 
However, finally a new meander starts to develop at the end of the year (lower panels). Similar detachments of eddies from Kuroshio meanders have been detected in another primitive-equation model and in in-situ observations (Qiu and Miao, 2000, their Figs. 9 and 10). Moreover, Fig. 4 indicates that the signatures of the different meander paths are also found in the deeper ocean which leads us to the hypothesis that the transition between both meander states is a barotropically controlled phenomenon.

\section{b. Bimodality of the Gulf Stream}

In Fig. 5a, the latitudinal position of the maximum zonal geostrophic velocity at $75^{\circ} \mathrm{W}$ is plotted over the period 1979-1997. During the first year, the maximum zonal geostrophic velocity is at a latitude of about $34.5^{\circ} \mathrm{N}$ and a contour plot of SSH anomalies is shown for January 1979 in Fig. 5b. Like the Kuroshio, the Gulf Stream also separates too far north compared to reality. It is characterized by a weak southern recirculation cell and a strong anti-cyclonic cell near Cape Hatteras, which is not observed in the North Atlantic Ocean. A similar mean state is also found in other high resolution models (Beckmann et al., 1994; Bryan et al., 1995; Maltrud et al., 1998). This pattern is quite similar to the observed Gulf Stream in its weakly deflected configuration (cf. Fig. 2), as far as the flow between the Florida Straits and Cape Hatteras is concerned, and we will refer to it as Gulf Stream state $G_{b}^{P}$. After a year, a significant shift southward occurs in the latitudinal position of the maximum zonal geostrophic velocity (Fig. 5a). The SSH anomalies for January 1981 (Fig. 5c) display a Gulf Stream which actually seems to separate twice. First, it separates too far south compared to reality, and later on it separates too far north (at about the same latitude as in Fig. 5b). It is characterized by a strong southern and a weak northern recirculation cell, centered at about $\left(74^{\circ} \mathrm{W}, 32^{\circ} \mathrm{N}\right)$ and $\left(73^{\circ} \mathrm{W}, 34^{\circ} \mathrm{N}\right)$, respectively, and again the strong unrealistic anti-cyclonic cell near Cape Hatteras. There are some differences between this pattern and the observed Gulf Stream in its strongly deflected configuration (cf. Fig. 2) in that the simulated path deflects farther to the north and that the amplitude of the associated meander is much larger than the observed one. We will refer to the simulated path of Fig. 5c as Gulf Stream state $G_{c}^{P}$. The southerly position of the maximum zonal geostrophic velocity is maintained for about two years and then the earlier position is retained (Fig. 5a).

Also in the Gulf Stream region (Figs. 5b and c), heat is strongly advected with the mean flow. The Gulf Stream advects more heat northward in the case of path $G_{b}^{P}$ (Fig. 5b) than in the case of path $G_{c}^{P}$ (Fig. 5c). At $75^{\circ} W$ the maximum temperature gradient, i.e. the cold wall, of Gulf Stream state $G_{b}^{P}$ is situated at about $35^{\circ} N$ (Fig. $5 \mathrm{~b}$ ), and the cold wall of Gulf Stream state $G_{c}^{P}$ has a more southerly position at about $34^{\circ} N$ (Fig. 5c), in correspondence with Fig. 5a. Analysis of the temperature fields at depth in POCM (Figs. 5d and e) indicates that the signatures of the different mean paths are also found in the deeper ocean which leads us to the hypothesis that the transition between both patterns is a barotropically controlled phenomenon, as was also hypothesized for the Kuroshio. 
To summarize, in the POCM results indications of bimodal behavior are found for both Kuroshio and Gulf Stream. The bimodality of the Kuroshio occurs on interannual timescales and switches in the latitudinal position of the maximum geostrophic velocity are about $2^{\circ}$. For the Gulf Stream region, only one of these transitions is found and the lateral excursion of the maximum geostrophic velocity is smaller. In the next sections, we are going to explore whether multiple steady states of the Kuroshio and the Gulf Stream are dynamically possible. To test the hypothesis that the transitions are barotropically controlled, a barotropic SW model is used.

\section{The barotropic shallow-water model}

This model is similar to that used in Schmeits and Dijkstra (2000) and hence only the essentials will be repeated.

\section{a. Formulation}

Consider a flat-bottomed ocean basin with a realistic horizontal domain, $\mathcal{V}$, and bounded by a closed contour $\Gamma$. The density of the ocean is constant and the flow is driven by a windstress $\tau(\phi, \theta)=\tau_{0}\left(\tau^{\phi}, \tau^{\theta}\right)$, where $\tau_{0}$ is the amplitude and $\left(\tau^{\phi}, \tau^{\theta}\right)$ provides the spatial pattern. Lateral friction, with lateral friction coefficient $A_{H}$, is the dissipative mechanism in the model. In the usual notation, the velocities in eastward and northward directions are indicated by $u$ and $v$, respectively and $h$ is the thickness of the water column (with equilibrium value $D$ ), and changes due to changes in the sea surface height. The governing shallow-water equations are non-dimensionalized using scales $r_{0}$, $D, U, r_{0} / U$ and $\tau_{0}$ for length, layer depth, velocity, time, and windstress, respectively, where $r_{0}$ is the radius of the earth, and become

$$
\begin{array}{r}
\epsilon\left(\frac{\partial u}{\partial t}+\frac{u}{\cos \theta} \frac{\partial u}{\partial \phi}+v \frac{\partial u}{\partial \theta}-u v \tan \theta\right)- \\
-v \sin \theta=-\frac{\epsilon F}{\cos \theta} \frac{\partial h}{\partial \phi}+E\left(\nabla^{2} u-\frac{u}{\cos ^{2} \theta}-\frac{2 \sin \theta}{\cos ^{2} \theta} \frac{\partial v}{\partial \phi}\right)+\alpha \frac{\tau^{\phi}}{h} \\
\epsilon\left(\frac{\partial v}{\partial t}+\frac{u}{\cos \theta} \frac{\partial v}{\partial \phi}+v \frac{\partial v}{\partial \theta}+u^{2} \tan \theta\right)+ \\
+u \sin \theta=-\epsilon F \frac{\partial h}{\partial \theta}+E\left(\nabla^{2} v-\frac{v}{\cos ^{2} \theta}+\frac{2 \sin \theta}{\cos ^{2} \theta} \frac{\partial u}{\partial \phi}\right)+\alpha \frac{\tau^{\theta}}{h} \\
\frac{\partial h}{\partial t}+\frac{1}{\cos \theta}\left(\frac{\partial(h u)}{\partial \phi}+\frac{\partial(h v \cos \theta)}{\partial \theta}\right)=0 .
\end{array}
$$

On the boundary $\Gamma$ of the domain no-slip conditions are prescribed; that is,

$$
(\phi, \theta) \varepsilon \Gamma: \quad u=v=0 .
$$


The parameters in these equations are the Rossby number $\epsilon$, the Froude number $F$, the Ekman number $E$, and the windstress coefficient $\alpha$. Expressions for these parameters are

$$
\epsilon=\frac{U}{2 \Omega r_{0}} ; F=\frac{g D}{U^{2}} ; E=\frac{A_{H}}{2 \Omega r_{0}^{2}} ; \alpha=\frac{\tau_{0}}{2 \Omega \rho D U},
$$

where $\Omega$ is the angular velocity of the earth. Standard values of the parameters in this model are listed in Table 1. Note that only the amplitude of the windstress (Trenberth et al., 1989) and therefore the value of the parameter $\alpha$ differs between the North Atlantic and North Pacific case.

\section{b. Numerical methods}

On each particular domain below, a finite difference discretization is used on a staggered grid. Continental geometry and boundary conditions are taken into account by first discretizing the equations on the sphere and then substituting equations with boundary conditions, according to whether the point is a land point or an ocean point. To ensure overall mass conservation, an integral condition for $h$ over the domain $\mathcal{V}$ is implemented (Schmeits and Dijkstra, 2000); that is,

$$
\int_{\mathcal{V}} h \cos \theta d \phi d \theta=|\mathcal{V}|
$$

where $|\mathcal{V}|$ is the (dimensionless) area of the domain since the layer depth is scaled with $D$.

The basic technique for dynamical systems analysis is comprised of two main pieces: a continuation method to advance one step on a branch of steady states as a parameter is varied, and an eigenvalue solver to determine the linear stability of the computed steady state (e.g. Dijkstra, 2000). Discretization of the steady-state solutions leads to a set of nonlinear algebraic equations of the form

$$
\mathbf{F}(\mathbf{u}, \mathbf{p})=0 \text {. }
$$

Here $\mathbf{u}$ is a $d$-dimensional vector consisting of the unknowns at the grid points, $\mathbf{p}$ is the $p$-dimensional vector of parameters, and $\mathbf{F}$ is a nonlinear mapping from $R^{d} \times R^{p} \rightarrow$ $R^{d}$, where $d$ indicates the number of degrees of freedom. To determine branches of steady solutions of the Eq. (5) as one of the parameters (say $\mu$ ) is varied, the pseudoarclength method is used. The branches $(\mathbf{u}(s), \mu(s))$ are parametrized by an "arclength" parameter $s$. An additional equation is obtained by "normalizing" the tangent

$$
\dot{\mathbf{u}}_{0}^{T}\left(\mathbf{u}-\mathbf{u}_{0}\right)+\dot{\mu}_{0}\left(\mu-\mu_{0}\right)-\Delta s=0,
$$

where $\left(\mathbf{u}_{0}, \mu_{0}\right)$ is an analytically known starting solution or a previously computed point on a particular branch and $\Delta s$ is the steplength. 
When a steady state is determined, the linear stability of the solution is considered and transitions that mark qualitative changes such as transitions to multiple equilibria (pitchfork or saddle node bifurcations) or periodic behavior (Hopf bifurcations) can be detected (e.g. Dijkstra, 2000). The linear stability analysis amounts to solving a generalized eigenvalue problem of the form

$$
\mathrm{Ax}=\sigma \mathrm{Bx}
$$

where $A$ and $B$ are nonsymmetric matrices. Bifurcations are detected from crossings of $\sigma$ with the imaginary axis. Solution techniques for these problems are presented in Dijkstra et al. (1995).

A nice spin-off of steady state solvers is the immediate availability of an implicit time integration scheme. Using a time step $\Delta t$, and a time index $n$, a class of two-level schemes can be written as

$$
\mathbf{M} \frac{\mathbf{u}^{n+1}-\mathbf{u}^{n}}{\Delta t}+\Theta \mathbf{F}\left(\mathbf{u}^{n+1}\right)+(1-\Theta) \mathbf{F}\left(\mathbf{u}^{n}\right)=0
$$

For $\Theta=1$, this is the Backward Euler scheme and for $\Theta=1 / 2$, it is the Crank-Nicholson scheme. The equations for $\mathbf{u}^{n+1}$ are solved by the Newton-Raphson technique and lead to large systems of nonlinear algebraic equations, similar to that for the steady state computation. It is well-known that the Crank-Nicholson scheme is unconditionally stable for linear equations. This does not mean that one can take any time step, since this quantity is still constrained by accuracy. Although the scheme is second order accurate in time, large discretization errors occur when the time step is too large. Second limitation on the time step is the convergence domain of the Newton-Raphson process, which does not necessarily converge for every time step.

\section{Bifurcation analysis of the barotropic shallow- water model}

In the results below, bifurcation diagrams are computed for steady wind forcing using the Ekman number as a control parameter, the latter being the most uncertain parameter in the model. Every point on a curve in such a bifurcation diagram corresponds to a stationary state and stability is indicated with the linestyle (drawn is stable and dotted is unstable). On the vertical axis, the maximum northward volume transport $\Phi$ (in $\mathrm{Sv}$ ) over a section is shown which is calculated as

$$
\Phi=\left(U D r_{0}\right) \max \int_{\phi_{W}}^{\phi} v h \cos \theta d \phi
$$

where the maximum is taken both over $\phi$ and $\theta$. All parameters except the Ekman number are fixed as in Table 1 and the North Atlantic and North Pacific cases differ only in the wind stress pattern and amplitude, the domain size, the basin geometry and slightly in resolution $\left(\frac{1}{2}^{\circ}\right.$ and $\frac{5}{12}^{\circ}$, respectively). 


\section{a. Multiple equilibria of the Kuroshio}

Part of the North Pacific basin $\left[120^{\circ} \mathrm{E}, 150^{\circ} \mathrm{W}\right] \times[10,55]^{\circ} \mathrm{N}$ is considered using a horizontal resolution of $\frac{5}{12}^{\circ} \times \frac{5}{12}^{\circ}$. We have taken the $100 \mathrm{~m}$ depth contour as the continental boundary because otherwise the modeled Kuroshio would enter the East China Sea. In reality, it is steered by bottom topography, so that it follows a more or less straight path from Taiwan to Japan. The spatial pattern of the climatological wind stress forcing (Trenberth et al., 1989) over the North Pacific domain is displayed in Fig. 6a which shows the predominantly easterly (westerly) winds in the lower (mid)latitudes.

The bifurcation diagram for the North Pacific domain (Fig. 7a) consists of a perturbed pitchfork bifurcation (Golubitsky and Schaeffer, 1985) and clearly shows that multiple equilibria exist when the lateral friction is small enough. Note that there is quite a range of Ekman numbers where two equilibria are (barotropically) stable. Down to $E=1.8 \times 10^{-7}$, there is a unique steady solution for each value of the Ekman number (Fig. 7a). An example of a stationary solution in this regime is shown as a contour plot of SSH for $E=3.0 \times 10^{-7}$ in Fig. $7 \mathrm{~b}$ over the whole computational domain. An inset of the flow pattern in the Kuroshio region is presented in Fig. 8a, where the colors indicate the magnitude of lateral friction (upper panel), $\beta$-effect (middle panel) and inertia (lower panel) in the barotropic potential vorticity equation (similar to the vorticity budget contour plots in the study of Özgökmen et al., 1997). The barotropic potential vorticity equation is derived from (1) by taking the curl of the momentum equations (1a) and (1b), and can schematically be written as:

$$
\frac{\partial \zeta}{\partial t}=-(I+B+F+W)=0
$$

where $\zeta$ is the relative vorticity, $I$ is inertia, $B$ the $\beta$-effect, $F$ lateral friction and $W$ the wind stress curl. The stationary solution at $E=3.0 \times 10^{-7}$ displays a Kuroshio path south of Japan, referred to as $K_{b}^{S}$ (Fig. 8a), quite similar to the observed small meander state (cf. Fig. 1). The modeled Kuroshio eventually separates too far north, compared to reality, but at about the same latitude as the simulated Kuroshio in POCM (cf. Fig. 3). At this value of $E$, the transport $\Phi$ is about $66 \mathrm{~Sv}$.

The upper branch of solutions in Fig. 7a continues to exist for values smaller than $E=1.8 \times 10^{-7}$, but the solutions loose stability at $E=1.2 \times 10^{-7}$. They become unstable to one oscillatory mode at a Hopf bifurcation $H_{1}$ and to another oscillatory mode at Hopf bifurcation $\mathrm{H}_{2}$ (Fig. 7a). The former oscillatory mode has a timescale of 2 months and has its maximum amplitude in the high shear region to the south of Kamchatka; the latter oscillatory mode has a timescale of 3 months and is located in the separation region of the Kuroshio, where the shear is also high. A stable stationary solution on this branch is shown for $E=1.5 \times 10^{-7}$, which is in the multiple equilibria regime, in Fig. 7c. It displays a Kuroshio path south of Japan, referred to as $K_{c}^{S}$ (Fig. 8b), different from both the observed and POCM meander states (cf. Figs. 1 and 3 ), with a transport $\Phi$ of about $86 \mathrm{~Sv}$. Compared to the solution at $E=3.0 \times 10^{-7}$ (Fig. 7b) to which it is continuously connected, the anti-cyclonic recirculation gyre to 
the south of Japan has intensified and has caused the Kuroshio to deviate from the coast. The western boundary layer at $E=1.5 \times 10^{-7}$ is inertially controlled to a large extent (Fig. 8b), whereas the boundary layer at $E=3.0 \times 10^{-7}$ is of the nonlinear Munk type (Fig. 8a).

The second branch of solutions exists only for $E<1.8 \times 10^{-7}$, which is the position of the saddle node bifurcation on this branch. Steady states on the upper part of the branch are stable down to $E=1.2 \times 10^{-7}$ and become unstable to the same oscillatory mode as the steady states on the upper branch at Hopf bifurcation $H_{1}$ (Fig. 7a). The solution at $E=1.5 \times 10^{-7}$ (Fig. 7 d) displays a Kuroshio path south of Japan, referred to as $K_{d}^{S}$ (Fig. 8c), quite similar to the observed and POCM large meander states (cf. Figs. 1 and 3c). It eventually separates at the southeast corner of Honshu, which corresponds to the observed separation point. At this value of $E$, the transport $\Phi$ is about $84 \mathrm{~Sv}$, which is about the same as that of $K_{c}^{S}$ (Fig. 7c).

The third steady state at $E=1.5 \times 10^{-7}$, referred to as $K_{e}^{S}$ (Fig. 7e), is similar to $K_{d}^{S}$ (Fig. $7 \mathrm{~d}$ ), but it is unstable and it has different final separation behavior. The western boundary layers of $K_{d}^{S}$ and $K_{e}^{S}$ are highly nonlinear, with lateral friction playing a minor role (Figs. 8c and d). Outside the western boundary layers the Sverdrup balance holds for all four steady state solutions. The circulation patterns outside the region of the western boundary current are very similar (Figs. 7c, d and e), so that the multiple equilibria are related to the different meandering structures of the Kuroshio. The transports for the stationary solutions in the multiple equilibria regime (Fig. 7a) are much larger than current estimates to the south of Japan of about 50-55 Sv (Qiu and Joyce, 1992). This overestimation of the transports is probably due to the neglect of bottom topography in the model.

\section{b. Multiple equilibria of the Gulf Stream}

In this case, the full North Atlantic basin $[85,5]^{\circ} W \times[10,65]^{\circ} \mathrm{N}$ is considered using a horizontal resolution of $\frac{1}{2}^{\circ} \times \frac{1}{2}^{\circ}$ as in Schmeits and Dijkstra (2000). The spatial pattern of the climatological wind stress forcing (Trenberth et al., 1989) over the North Atlantic domain is plotted in Fig. 6b, showing similar features as over the North Pacific domain.

The bifurcation diagram for the North Atlantic domain is shown in Fig. 9a. The diagram again consists of a perturbed pitchfork bifurcation and clearly shows that multiple equilibria exist when the lateral friction is small enough, similar to the North Pacific case. However, there does not exist a range of Ekman numbers where the multiple equilibria are (barotropically) stable. Because of the minor difference in resolution, the bifurcation diagram for the North Atlantic domain would change only quantitatively but not qualitatively if we would increase the resolution to the same resolution as used for the North Pacific domain, i.e. $\frac{5}{12}^{\circ}$. In other words, the same bifurcation points would still be present, but they would probably shift a bit.

Two solution branches are found; down to $E=2.2 \times 10^{-7}$, there is only one branch of solutions (Fig. 9a). Steady states on this branch are stable down to $E=2.5 \times 10^{-7}$ and become unstable to one oscillatory mode at the Hopf bifurcation $H_{1}$ (Fig. 9a). 
This oscillatory mode has been linked (Schmeits and Dijkstra, 2000) to intermonthly variability of the Gulf Stream as found in earlier studies (Lee and Cornillon, 1995).

A stationary solution on this lower branch in Fig. 9a is shown as a contour plot of SSH for $E=1.6 \times 10^{-7}$ in Fig. 9b. It displays a Gulf Stream path, referred to as $G_{b}^{S}$ (Fig. 10a), surprisingly similar to Gulf Stream state $G_{b}^{P}$ in POCM (cf. Fig.5b), with a transport $\Phi$ of about $46 \mathrm{~Sv}$. This solution resembles also the 5-year mean plot of upperlayer transport streamfunction for experiment V of Özgökmen et al. (1997) (their Fig. 5a), who have integrated a two-layer QG model (including both quasi-realistic bottom topography and continental geometry), driven by a single-gyre wind forcing. The other branch in Fig. 9a exists only for $E<2.2 \times 10^{-7}$, which is the position of the saddle node bifurcation. A second Hopf bifurcation $\left(\mathrm{H}_{2}\right)$ occurs on this branch (Fig. 9a) and the period of the oscillatory mode is 2 months. The maximum amplitude of the unstable mode is found in the high shear region to the southeast of Greenland (Schmeits and Dijkstra, 2000).

The solution at $E=1.6 \times 10^{-7}$ on the lower part of the upper branch (Fig. 9c) displays a Gulf Stream path, referred to as $G_{c}^{S}$ (Fig. 10b), quite similar to the observed strongly deflected Gulf Stream (cf. Fig. 2). However, the Gulf Stream in the SW model, like the real Gulf Stream, deflects further upstream than Gulf Stream state $G_{c}^{P}$ in POCM (cf. Fig.5c), and the amplitude of the meander associated with Gulf Stream state $G_{c}^{S}$ in the SW model is too large compared with the real Gulf Stream. At this value of $E$, the transport $\Phi$ is about $70 \mathrm{~Sv}$, which is 1.5 times larger than that in Fig. $9 \mathrm{~b}$, and somewhat larger than current estimates near Cape Hatteras of about 50-65 Sv (Johns et al., 1995). The third steady state at $E=1.6 \times 10^{-7}$, referred to as $G_{d}^{S}$ (Fig. 9d), is similar to Gulf Stream solution $G_{c}^{S}$ (Fig. 9c), but has hardly any cyclonic recirculation cell. The circulation patterns outside the region of the western boundary current are very similar again (Figs. 9b, c and d), so that the multiple equilibria are related to the different structures of the Gulf Stream. Outside the western boundary layers the Sverdrup balance holds for all three steady state solutions. For Gulf Stream solution $G_{b}^{S}$, the boundary layer is of the nonlinear Munk type (Fig. 10a). However, despite the similarity in flow patterns, the vorticity balance in the western bounday layer of experiment V of Özgökmen et al. (1997) (their Fig. 7) is mainly inertially controlled. On the other hand, for Gulf Stream solutions $G_{c}^{S}$ and $G_{d}^{S}$ the boundary layer is highly nonlinear, with lateral friction playing a minor role (Figs. 10b and c).

\section{$5 \quad$ Noise-induced transitions}

The bifurcation diagrams in the previous section immediately indicate the parameter range in Ekman number where transitions between steady states can occur. In this section, transient flows are computed with the same SW model. Using implicit time integration, both steady and noisy wind forcing are employed to determine under which conditions transitions occur. 


\section{a. Stochastic windstress forcing}

The stochastic wind stress forcing has been derived from the monthly mean NCEP Reanalysis $10 \mathrm{~m}$ wind data for the period from 1949-1999 (i.e. 612 months). These wind data have been converted to wind stress data using a drag coefficient of $1.0 \times 10^{-3}$. Besides, the climatological monthly means have been subtracted from the data to form anomalies. The highest variability of the zonal wind stress anomalies in the North Atlantic (Fig. 11a) is to the south of Iceland where the amplitude of the mean forcing is also high (Fig. 6b). In the North Pacific there are several locations with relatively high variability (Fig. 11a). As a measure of the "noisiness" of the data (Blanke et al., 1997) we have plotted the one-month-lag autocorrelation of the zonal wind stress anomalies in Fig. 11b. In the North Atlantic and North Pacific basins the autocorrelation is generally less than 0.2 , except in the lowest latitudes.

Figs. 11c-d show analogous statistics for meridional stress anomalies. The highest variability in the North Atlantic domain occurs to the southwest of Iceland and to the east of Cuba, and the highest variability in the North Pacific domain occurs to the north of Hawaii (Fig. 11c). As was the case for the zonal wind stress anomalies, the one-month-lag autocorrelation of the meridional wind stress anomalies is generally less than 0.2 , except in the lowest latitudes (Fig. 11d). We conclude therefore that the wind stress anomalies can be used as an uncorrelated noise product. As a phenomenon with short decorrelation time has approximately a flat spectrum at frequencies lower than the inverse of the decorrelation time, we can approximate the stochastic forcing as white on timescales longer than a month. The detailed decorrelation structure of weather noise in timescales shorter than a month has been ignored, on the assumption that this will not matter to the interannual behavior (Blanke et al., 1997).

The maximum amplitude of the wind stress anomalies (0.16 $\mathrm{Pa}$ for both basins) is of the same order of magnitude as the maximum amplitude of the climatological mean forcing (cf. Table 1). Based on the findings of others (e.g. Willebrand et al., 1980) that observed stochastic forcing produces a primarily barotropic response, it seems justified to use a barotropic ocean model in order to study the effect of stochastic forcing. The stochastic forcing has been implemented in the model as follows. The monthly stochastic forcing is defined by entire wind stress anomaly fields chosen randomly among the 612month sample in order to preserve the intrinsic spatial coherence of the fields, similar to Blanke et al. (1997).

In all time integrations, described below, the SW model has been run for 6-9 years with the standard set of parameters (Table 1) apart from the value of the Ekman number. Since we are interested to monitor whether transitions between different steady states occur, the spatial rms value of the difference between the instantaneous field $h^{W B C}$ and each steady state field $\bar{h}_{i}^{W B C}(i=1,2,3)$, called $R_{i}$, is computed in either the Gulf Stream region $[85,70]^{\circ} W \times[25,40]^{\circ} N$ or the Kuroshio region $[120,150]^{\circ} E \times[25,40]^{\circ} N$, as follows:

$$
R_{i}=\frac{D\left|\left(h^{W B C}-\bar{h}_{i}^{W B C}\right)\right|_{2}}{\sqrt{N}}
$$


Here, $|\cdot|_{2}$ corresponds to the $\mathrm{L}^{2}$-norm, and $N$ is the total number of grid points in the Gulf Stream or Kuroshio region.

\section{b. Transitions between different paths of the Gulf Stream}

For $E=1.6 \times 10^{-7}$, which is a value in the multiple equilibria regime for the North Atlantic basin (Fig. 9a), two trajectories were computed. As initial condition the unstable steady solution $G_{b}^{S}$ (Fig. 9b) indicated by the point b in Fig. 9a was chosen which has been slightly perturbed into the direction of the most unstable mode in the case of the climatological mean wind forcing.

For the first trajectory, the flow is forced by the climatological mean wind forcing, there is no noise added and values of the maximum northward transport are shown in Fig. 12a. After an initial transient period, eventually the trajectory is attracted towards a stable limit cycle with a period of about two months, coming from the second Hopf bifurcation in Fig. 9a. The mean SSH pattern associated with the stable limit cycle is shown in Fig. 12b; it is very similar to state $G_{c}^{P}$ in POCM (cf. Fig. 5c).

For the second trajectory, the stochastic forcing is added to the climatological mean forcing and values of $R_{i}$ are shown in Fig. 13a. The correspondence between the value of $i$ and the steady states is indicated in the figure caption. The trajectory stays more or less close to the unstable steady solution from which it started $\left(G_{b}^{S}\right.$; indicated by point b in Fig. 9a) during the first five years of the integration, but it is attracted towards one of the other unstable steady solutions $\left(G_{c}^{S}\right.$; indicated by point c in Fig. 9a) in the last part of the integration. At $t=0.61 \mathrm{yr}$, the trajectory is farthest away from any of the unstable steady state solutions at $E=1.6 \times 10^{-7}$ (Fig. 13a) and a snapshot of $\mathrm{SSH}$ at this time is shown in Fig. 13b. The Gulf Stream penetrates into the basin to some extent and there are nicely developed northern and southern recirculation gyres. At $t=0.73 \mathrm{yr}$, the trajectory is still far from any of the unstable steady state solutions (Fig. 13a), but the SSH pattern (Fig. 13c) is quite different from that shown in Fig. 13b. At this time, the Gulf Stream is similar to the pattern associated with the limit cycle in the SW model (Fig. 12b) and to state $G_{c}^{P}$ in POCM (cf. Fig. 5c).

At $t=4.44 \mathrm{yr}$, the trajectory is quite close to the unstable steady solution $G_{b}^{S}$ (Fig. 9b), from which it started. This is confirmed by the SSH pattern (Fig. 13d) and the value of $R_{1}$ which is only $2.1 \mathrm{~cm}$ at this time (Fig. 13a). A substantial transition in the flow is observed near $t=4.8 \mathrm{yr}$, with the value of $R_{1}\left(R_{3}\right)$ increasing (decreasing) rapidly. At $t=5.82 \mathrm{yr}$, the trajectory is the closest to the unstable steady solution $G_{c}^{S}$ (Fig. 9c), which is again confirmed by the SSH pattern at this time (Fig. 13e), with a $R_{3}$ value of only $1.6 \mathrm{~cm}$ (Fig. $13 \mathrm{a}$ ).

\section{c. Transitions between different paths of the Kuroshio}

For the North Pacific domain, a value of $E=1.1 \times 10^{-7}$ was chosen, which is in the multiple equilibria regime (Fig. 7a). As initial condition, the unstable steady solution on the upper branch of Fig. 7a, with a pattern similar to that of $K_{c}^{S}$ (Fig. 7c), was 
chosen. Again, this state is slightly perturbed into the direction of the most unstable mode in the case of the climatological mean wind forcing.

For the first trajectory, the flow is forced by the climatological mean wind forcing and there is no noise added. Values of the maximum northward transport are shown in Fig. 14a. The maximum northward transport varies between 81 and $95 \mathrm{~Sv}$, which is the same range of transports as for the multiple steady states at $E=1.1 \times 10^{-7}$ (cf. Fig. 7a). However, it turns out that the trajectory stays relatively close to the unstable steady solution from which it started. There are no transitions to states similar to the other steady state solutions in Fig. 7a. In Fig. 14b the mean SSH field over the last year of the integration is plotted, the pattern being quite similar to the starting solution.

For the second trajectory, the stochastic forcing is added to the climatological mean forcing and values of $R_{i}$ are shown in Fig. 15a. In this case, the trajectory stays also relatively close to the unstable steady solution from which it started. For another trajectory, again with the additive stochastic forcing but using a smaller value of the Ekman number $\left(E=7.3 \times 10^{-8}\right)$, also no transitions in the values of $R_{i}$ are found (Fig. 15b). Several other trajectories have been computed for different values of $E$, but none of them shows transitions similar to those in the Gulf Stream. Although one cannot exclude that with carefully chosen initial conditions and longer time integrations one might find these transitions, it is remarkable that the noise in the wind stress over the Pacific is not able to induce robust transitions between steady states in the multiple equilibria regime.

\section{Discussion}

The results in the previous section raise an interesting issue of non-correspondence of the intermediate model results with observations and high resolution OGCM results. Whereas a clear transition is found between steady states in the Gulf Stream, bimodality is not obvious in observations. On the other hand, there is plenty of observational evidence for bimodality in the Kuroshio, but transitions between steady states in the Kuroshio are hard to find, even in a parameter regime where dynamically multiple states are allowed. What can be learned from this?

Although baroclinic effects and bottom topography are neglected, the intermediate complexity model cannot be rejected a priori to be able to describe the main structure of flow patterns associated with both western boundary currents. For the Gulf Stream, there is a close correspondence between the steady state $G_{b}^{S}$ in the intermediate complexity model (Fig. 10a) and the Gulf Stream pattern $G_{b}^{P}$ in POCM (Fig. 5b) and between the mean state of the limit cycle in the intermediate complexity model (Fig. 12b) and the Gulf Stream pattern $G_{c}^{P}$ in POCM (Fig. 5c). For the Kuroshio, the correspondence between the steady states in the intermediate complexity model (Figs. 8b-d) and the meander states in POCM (Figs. 3b-c) is also reasonable, although less good as for the Gulf Stream.

The results in Qiu and Miao (2000) are qualitatively consistent with the bifurcation 
diagram in Fig. 7a. For a realistic value of the wind stress amplitude, variations between a straight path and a meander path have been found on interannual timescales (Qiu and Miao, 2000, their Fig. 7), whereas the Kuroshio stays in the straight path state after an initial period of adjustment when the wind stress forcing is reduced by 25\% (Qiu and Miao, 2000, their Fig. 14). The model run with the realistic value of the wind stress amplitude (and a value of the lateral friction coefficient of $800 \mathrm{~m}^{2} \mathrm{~s}^{-1}$, which corresponds to a value of the Ekman number of $1.35 \times 10^{-7}$ in our SW model) is probably in the multiple equilibria regime (consistent with Fig. 7a), whereas the model run with reduced wind stress amplitude (equivalent with a larger value of the Ekman number in our SW model) is probably in that part of parameter space where there is only one state, i.e. the straight path state (consistent with Fig. 7a). This leads us to the view that the bimodality of the Kuroshio in the results of Qiu and Miao (2000) is also due to the existence of multiple equilibria. This contrasts their view, namely that the bimodality is a result of a self-sustained internal oscillation, which would imply the existence of a Hopf bifurcation, at which an interannual mode destabilizes.

The bifurcation diagram in Fig. 7a is also qualitatively consistent with analyses of observed sea level data by Kawabe (1995). He has concluded that the Kuroshio always takes a non-large meander path (i.e. is in the single equilibrium regime) for small transport, i.e. small amplitude of the forcing (equivalent with a relatively large Ekman number in our SW model), but can take either a large meander path or a non-large meander path (i.e. is in the multiple equilibria regime) for large and medium transports (corresponding to a relatively small Ekman number in our SW model).

The structure of multiple equilibria is also a robust dynamical property of the barotropic model. The existence of the multiple equilibria in the barotropic SW model with realistic geometry and forcing can be traced back to its basics (Dijkstra and Molemaker, 1999) as a spontaneous symmetry breaking. This symmetry breaking was analysed in the highly idealized case in a rectangular basin with symmetric wind stress forcing (Cessi and Ierley, 1995) and the associated physical mechanism (Dijkstra and Katsman, 1997) involves an asymmetric weakening/strengthening of the subpolar/subtropical gyre with a positive feedback from the resulting change in horizontal shear. The multiple equilibria in the simple case deform into solutions with different separation behavior of the Gulf Stream for the realistic case and no new equilibria are introduced by the change in geometry (Dijkstra and Molemaker, 1999). The results here, in particular the correspondence between the patterns of the different states, indicate that the multiple equilibria persist in a state-of-the-art ocean model, such as POCM.

Having the multiple equilibria in both western boundary currents, the presence of noise in the wind stress forcing is able to induce a transition in the Gulf Stream (Fig. 13a) whereas it is unable to induce transitions in the Kuroshio (Fig. 15). That the spatial structure of the noise is important has already be pointed out in Sura et al. (2001). They show that regime transitions are induced by the inhomogeneity of the white noise variance. Indeed, the variance of the wind stress forcing over the North Atlantic basin is spatially much more inhomogeneous than that of the wind stress forcing over the 
North Pacific basin (Fig. 11a).

If multiple steady states are indeed the underlying cause of the bimodality of the Kuroshio, then there must be physical effects, not considered in the barotropic SW model, which destabilize the Kuroshio mean flow. Simultaneously, similar or additional effects neglected must stabilize the flow in the Gulf Stream area, because actual observed bimodality is weak. Stratification can certainly influence the mean flow through pressure gradients, and additionally instabilities, i.e. baroclinic eddies, can be generated that may cause a strong rectification of the mean state. Baroclinic instability is a major element in the mechanism of transition from the straight to the meander path suggested in Qiu and Miao (2000), but transitions between both paths have also been found in barotropic models (e.g. Chao, 1984). Moreover, there seems to be no reason why the baroclinic effects would differ substantially between the Gulf Stream and Kuroshio. A second effect neglected, namely bottom topography, is substantially different in both regions and this seems to be one of the possibilities to explain the different behavior of the western boundary currents. In a two-layer context, bottom topography does not change the steady state solutions as presented for the barotropic case, since the bottom layer is always motionless in steady state. However, when time integrations are performed, bottom topographic features in the Kuroshio region may induce transitions between the steady states, whereas bottom topography in the Gulf Stream region may prohibit transitions. As it has a large effect on the sea surface height in a barotropic model, bottom topography was not incorporated here. While one also cannot exclude the effect of coupled ocean-atmosphere interaction on the stability of the flows, another important stabilizing mechanism in the North Atlantic may be the presence of the strong deep western boundary current (DWBC), which has been shown to fix the separation point in an idealized model context (Tansley and Marshall, 2000). The DWBC in the North Pacific is much weaker and is shielded from the southern coast of Japan by the Izu Ridge.

The results here also put the difficulties of the separation of the western boundary currents in high resolution ocean models (Özgökmen et al., 1997; Chassignet et al., 2001; Smith et al., 2000) in a different perspective. Our results suggest that the problems may not only be related to errors in representation of topography, forcing or friction, but substantial complexity is introduced through the nonlinear effects in the western boundary current region. In the SW model the Gulf Stream solutions $G_{c}^{S}$ and $G_{d}^{S}$ (Figs. 10b-c) and the Kuroshio in the multiple equilibria regime (Figs. 8b-d) are to a large extent inertially controlled, whereas Gulf Stream solution $G_{b}^{S}$ (Fig. 10a) and Kuroshio's meander state $K_{b}^{S}$ (Fig. 8a) are more viscously controlled. A delicate balance in the western boundary region may therefore determine which type of path will be present during a certain time interval of the simulation.

There may be a concern with respect to the resolution of the viscous sublayers of the multiple steady state solutions (Figs. 8b-d and 10a-c), as Smith et al. (2000) have suggested that inadequate resolution of the Munk layer can lead to changes in the Gulf Stream behavior. However, having the constraint that we cannot easily repeat the calculations at much higher resolution, we think that the results on bimodality are 
robust, even in the regime of small Ekman numbers considered. This is based on the robustness of the (im)perfect pitchfork (and hence of the multiple equilibria) structure in a hierarchy of models, as mentioned above. This hierarchy includes models with very high resolution in the western boundary layer, such that the viscous sublayer is accurately resolved. Therefore, we think that the remark of Smith et al. (2000) about the regime transitions they found in the Gulf Stream during the spinup phase of the POP model supports our view, i.e. that there are multiple steady states in the Gulf Stream, at least in numerical models and even on a truly eddy-resolving resolution $\left(\frac{1}{10}^{\circ}\right)$.

At this point, it is still difficult to relate the different amounts of northward heat transport associated with the different paths in POCM to that in reality. The meridional heat transport associated with Kuroshio's small meander state $K_{b}^{P}$ (Fig. 3b) may be larger than that of Kuroshio's large meander state $K_{c}^{P}$ (Fig. 3c) because of the differences in meridional heat advection. An analogous argument holds for Gulf Stream patterns $G_{b}^{P}$ (Fig. 5b) and $G_{c}^{P}$ (Fig. 5c), respectively. However, the Kuroshio (Gulf Stream) path in POCM is still quite different from the observed time mean Kuroshio (Gulf Stream) path, which penetrates far more into the North Pacific (Atlantic). Hence, the heat transport computed with POCM likely underestimates the actual changes due to a transition in the Kuroshio (Gulf Stream) path. Very high resolution simulations with (improved) ocean models, yielding more realistic Kuroshio (Gulf Stream) paths (e.g. Chassignet et al., 2001; Smith et al., 2000), and longer time series of high resolution data from observations are needed to shed more light on the changes in heat transport associated with the different meandering (deflection) paths. 


\section{Acknowledgments}

This work was supported by the Netherlands Organization for Scientific Research (NWO) under a PIONIER grant to HD. All computations were performed on the CRAY C90 at the Academic Computing Centre (SARA), Amsterdam, the Netherlands within the project SC498. Use of these computing facilities was sponsored by the National Computing Facilities Foundation with financial support from NWO. The authors are grateful to Robin Tokmakian (Naval Postgraduate School, Monterey, California, USA) for providing the POCM output. 


\section{References}

Auer, S. J., 1987: Five-year climatological survey of the Gulf Stream system and its associated rings. J. Geophys. Res., 92, $11709-11726$.

Bane, J. M. and W. K. Dewar, 1988: Gulf Stream bimodality and variability downstream of the Charleston bump. J. Geophys. Res., 93, 6695-6710.

Beckmann, A. C. W., C. W. Böning, J. Köberle, and J. Willebrand, 1994: Effects of increased horizontal resolution in a simulation of the North-Atlantic ocean. J. Phys. Oceanogr., 24, 326-344.

Blanke, B., J. D. Neelin, and D. Gutzler, 1997: Estimating the effect of stochastic wind stress forcing on ENSO irregularity. J. Climate, 10, 1473-1486.

Bryan, F. O., C. W. Böning, and W. R. Holland, 1995: On the midlatitude circulation in a high-resolution model of the North-Atlantic. J. Phys. Oceanogr., 25, 289-305.

Cessi, P. and G. R. Ierley, 1995: Symmetry-breaking multiple equilibria in quasigeostrophic, wind-driven flows. J. Phys. Oceanogr., 25, 1196-1205.

Chao, S.-Y., 1984: Bimodality of the Kuroshio. J. Phys. Oceanogr., 14, 92-103.

Charney, J. G. and G. R. Flierl, 1981: Ocean analogues of large-scale atmospheric motions. In Evolution of Physical Oceanography, Warren, B. and Wunsch, C., editors. The MIT Press, 504-548.

Chassignet, E. P., Z. D. Garraffo, R. D. Smith, and H. E. Hurlburt, 2001: High resolution Gulf Stream modeling. Submitted to Geophys. Res. Letters.

Dengg, J., A. Beckmann, and R. Gerdes, 1996: The Gulf Stream separation problem. In The warmwatersphere of the North Atlantic Ocean, Kraus, W. A., editor. Borntraeger, 253-290.

Dijkstra, H. A., 2000: Nonlinear Physical Oceanography. Kluwer Academic Publishers, $456+$ xviii pp.

Dijkstra, H. A. and C. A. Katsman, 1997: Temporal variability of the wind-driven quasigeostrophic double gyre ocean circulation: Basic bifurcation diagrams. Geophys. Astrophys. Fluid Dyn., 85, 195-232.

Dijkstra, H. A. and M. J. Molemaker, 1999: Imperfections of the North-Atlantic winddriven ocean circulation: continental geometry and windstress shape. J. Mar. Res., $57,1-28$.

Dijkstra, H. A., M. J. Molemaker, A. J. Van der Ploeg, and E. F. F. Botta, 1995: An efficient code to compute nonparallel flows and their linear stability. Comp. Fluids, 24, 415-434. 
Dijkstra, H. A., M. J. Schmeits, and C. A. Katsman, 1999: Internal variability of the North Atlantic wind-driven ocean circulation. Surveys in Geophysics, 20, 463-503.

Golubitsky, M. and D. Schaeffer, 1985: Singularities and groups in bifurcation theory. Springer-Verlag, 533 pp.

Hellerman, S. and M. Rosenstein, 1983: Normal monthly wind stress over the world ocean with error estimates. J. Phys. Oceanogr., 13, 1093-1104.

Hurlburt, H. E. and P. J. Hogan, 2000: Impact of $1 / 8^{\circ}$ to $1 / 64^{\circ}$ resolution on Gulf Stream model-data comparisons in basin-scale subtropical Atlantic Ocean models. Dyn. Atmos. Ocean., 32, 283-329.

Hurlburt, H. E., A. J. Wallcraft, W. J. Schmitz, P. J. Hogan, and E. J. Metzger, 1996: Dynamics of the Kuroshio/Oyashio current system using eddy-resolving models of the North Pacific Ocean. J. Geophys. Res., 101, 941-976.

Jiang, S., F. F. Jin, and M. Ghil, 1995: Multiple equilibria and aperiodic solutions in a wind-driven double gyre, shallow water model. J. Phys. Oceanogr., 25, 764-786.

Johns, W. E., T. J. Shay, J. M. Bane, and D. R. Watts, 1995: Gulf Stream structure, transport and recirculation near $68^{\circ}$ W. J. Geophys. Res., 100, 817-838.

Kawabe, M., 1986: Transition processes between the three typical paths of the Kuroshio. J. Oceanogr. Soc. Japan, 42, 174-191.

Kawabe, M., 1995: Variations of current path, velocity, and volume transport of the Kuroshio in relation with the large meander. J. Phys. Oceanogr., 25, 3103-3117.

Lee, T. and P. Cornillon, 1995: Temporal variation of meandering intensity and domainwide lateral oscillations of the Gulf Stream. J. Geophys. Res., 100(C7), 13,60313,613 .

Legeckis, R. V., 1976: The influence of bottom topography on the path of the Gulf Stream at latitude $31 \mathrm{~N}$ from NOAA's satellite imagery (abstract). Eos Trans. AGU, $57,260$.

Maltrud, M. E., R. D. Smith, A. J. Semtner, and R. C. Malone, 1998: Global eddyresolving ocean simulations driven by 1985-1995 atmospheric winds. J. Geophys. Res., 103, 30825-30853.

Masuda, A., 1982: An interpretation of the bimodal character of the stable Kuroshio path. Deep-Sea Res., 29, 471-484.

McWillams, J. C., 1996: Modeling the ocean general circulation. Ann. Rev. Fluid Mechanics, 28, 215-248. 
Olson, D. B., O. B. Brown, and S. R. Emmerson, 1983: Gulf Stream frontal statistics from Florida Straits to Cape Hatteras derived from satellite and historical data. J. Geophys. Res., 88, 4569-4577.

Özgökmen, T. M., E. P. Chassignet, and A. M. Paiva, 1997: Impact of wind forcing, bottom topography, and inertia on midlatitude jet separation in a quasigeostrophic model. J. Phys. Oceanogr., 27, 2460-2476.

Qiu, B. and T. M. Joyce, 1992: Interannual variability in the mid- and low-latitude western North Pacific. J. Phys. Oceanogr., 22, 1062-1079.

Qiu, B. and W. Miao, 2000: Kuroshio path variations south of Japan: bimodality as a self-sustained internal oscillation. J. Phys. Oceanogr., 30, 2124-2137.

Schmeits, M. J. and H. A. Dijkstra, 2000: Physics of the 9-month variability in the Gulf Stream region: combining data and dynamical systems analyses. J. Phys. Oceanogr., 30, 1967-1987.

Semtner, A. J. and R. M. Chervin, 1992: Ocean general circulation from a global eddy-resolving model. J. Geophys. Res., 97, 5493-5550.

Smith, R. D., M. E. Maltrud, F. O. Bryan, and M. W. Hecht, 2000: Numerical simulation of the North Atlantic Ocean at $\frac{1}{10}^{\circ}$. J. Phys. Oceanogr., 30, 1532-1561.

Speich, S., H. Dijkstra, and M. Ghil, 1995: Successive bifurcations in a shallow-water model applied to the wind-driven ocean circulation. Nonl. Proc. Geophys., 2, 241-268.

Stammer, D., R. Tokmakian, A. Semtner, and C. Wunsch, 1996: How well does a 1/4 global circulation model simulate large-scale oceanic observations? J. Geophys. Res., 101, 25779-25811.

Sura, P., K. Fraedrich, and F. Lunkeit, 2001: Regime transitions in a stochastically forced double-gyre model. J. Phys. Oceanogr., 31, 411-426.

Taft, B. A., 1972: Characteristics of the flow of the Kuroshio south of Japan. In Kuroshio, physical aspects of the Japan current, Stommel, H. and Yoshida, K., editors. University of Washington Press, 165-214.

Tansley, C. E. and D. P. Marshall, 2000: On the influence of bottom topography and the Deep Western Boundary Current on Gulf Stream separation. J. Mar. Res., 58, $297-325$.

Trenberth, K., J. Olson, and W. Large, 1989: A global ocean wind stress climatology based on ECMWF analyses. Technical report, National Center for Atmospheric Research, Boulder, Colorado.

Willebrand, J., S. G. H. Philander, and R. C. Pacanowski, 1980: The oceanic response to large-scale atmospheric disturbances. J. Phys. Oceanogr., 10, 411-429. 


\begin{tabular}{|c|c|c|c|}
\hline Dimensional parameters & \multicolumn{4}{|c|}{} \\
\hline Parameter & Value & Parameter & Value \\
\hline$r_{0}$ & $6.37 \times 10^{6} \mathrm{~m}$ & $\tau_{0}$ & $0.15(\mathrm{NA}) / 0.13(\mathrm{NP}) \mathrm{Pa}$ \\
\hline$D$ & $1.0 \times 10^{3} \mathrm{~m}$ & $A_{H}$ & $2.0 \times 10^{2} \mathrm{~m}^{2} \mathrm{~s}^{-1}$ \\
\hline$g$ & $9.8 \mathrm{~ms}^{-2}$ & $U$ & $1.0 \times 10^{-1} \mathrm{~ms}^{-1}$ \\
\hline$\rho$ & $1.0 \times 10^{3} \mathrm{kgm}^{-3}$ & $\Omega$ & $7.3 \times 10^{-5} \mathrm{~s}^{-1}$ \\
\hline Dimensionless parameters & Value & Parameter & Value \\
\hline Parameter & $1.0(N A) / 0.88(N P) \times 10^{-2}$ & $E$ & $3.4 \times 10^{-8}$ \\
\hline$\alpha$ & $1.1 \times 10^{-4}$ & $F$ & $9.8 \times 10^{5}$ \\
\hline$\epsilon$ &
\end{tabular}

Table 1: Standard values of parameters in the barotropic $S W$ model. NA refers to North Atlantic and NP to North Pacific. 

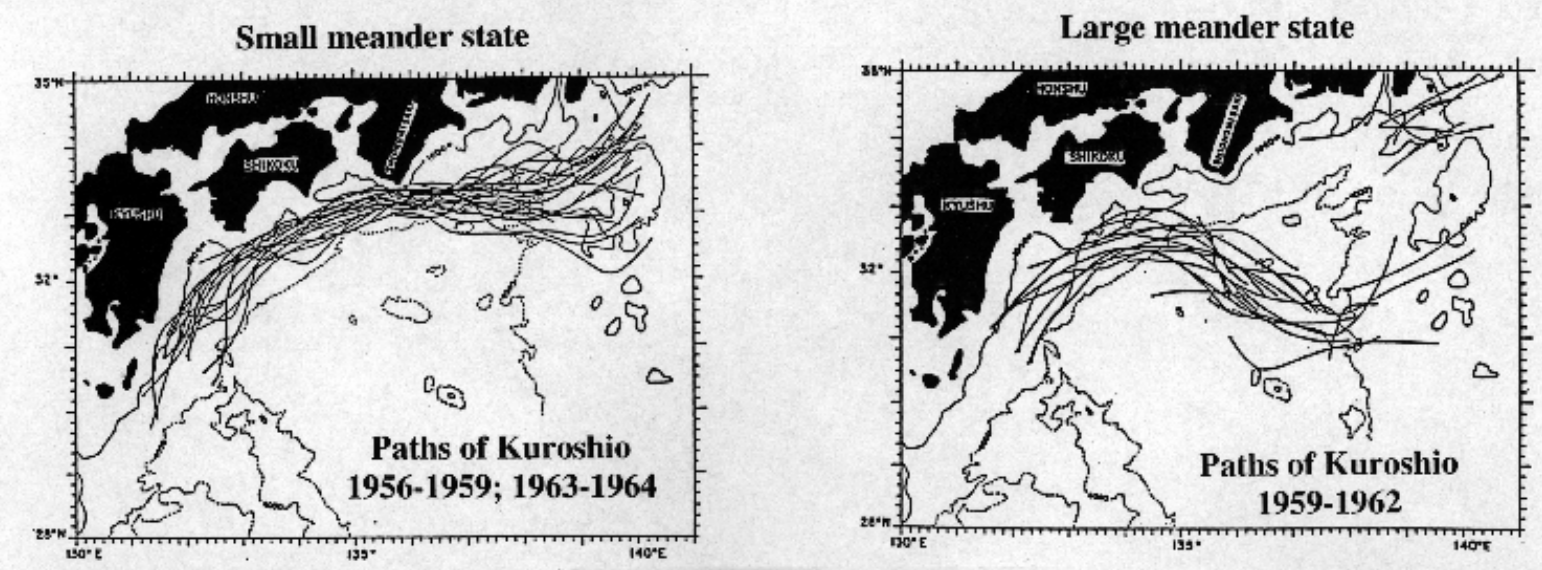

Figure 1: Examples of paths of the Kuroshio in its small meander state (left panel) and in its large meander state (right panel). The $1000 \mathrm{~m}$ (solid) and $4000 \mathrm{~m}$ (dotted) contours are also shown. (Adapted from Taft, 1972). 

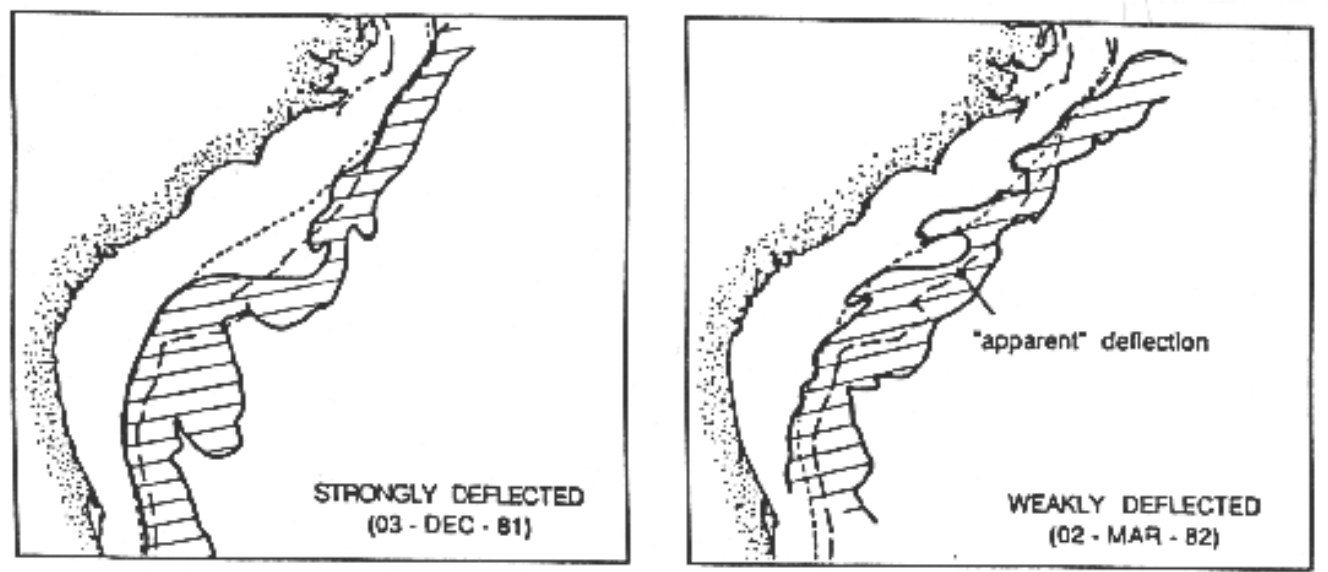

,...200 meter contour

--600 meter contour

Figure 2: Locations of the Gulf Stream on December 3, 1981, and March 2, 1982, during the Gulf Stream Deflection and Meander Energetics Experiment (DAMEX). These retraced versions of the AVHRR SST images from those dates show the stream in a typical strongly deflected configuration (December 3) and weakly deflected configuration (March 2) in the region of the Charleston bump. (From Bane and Dewar, 1988). 
(a)
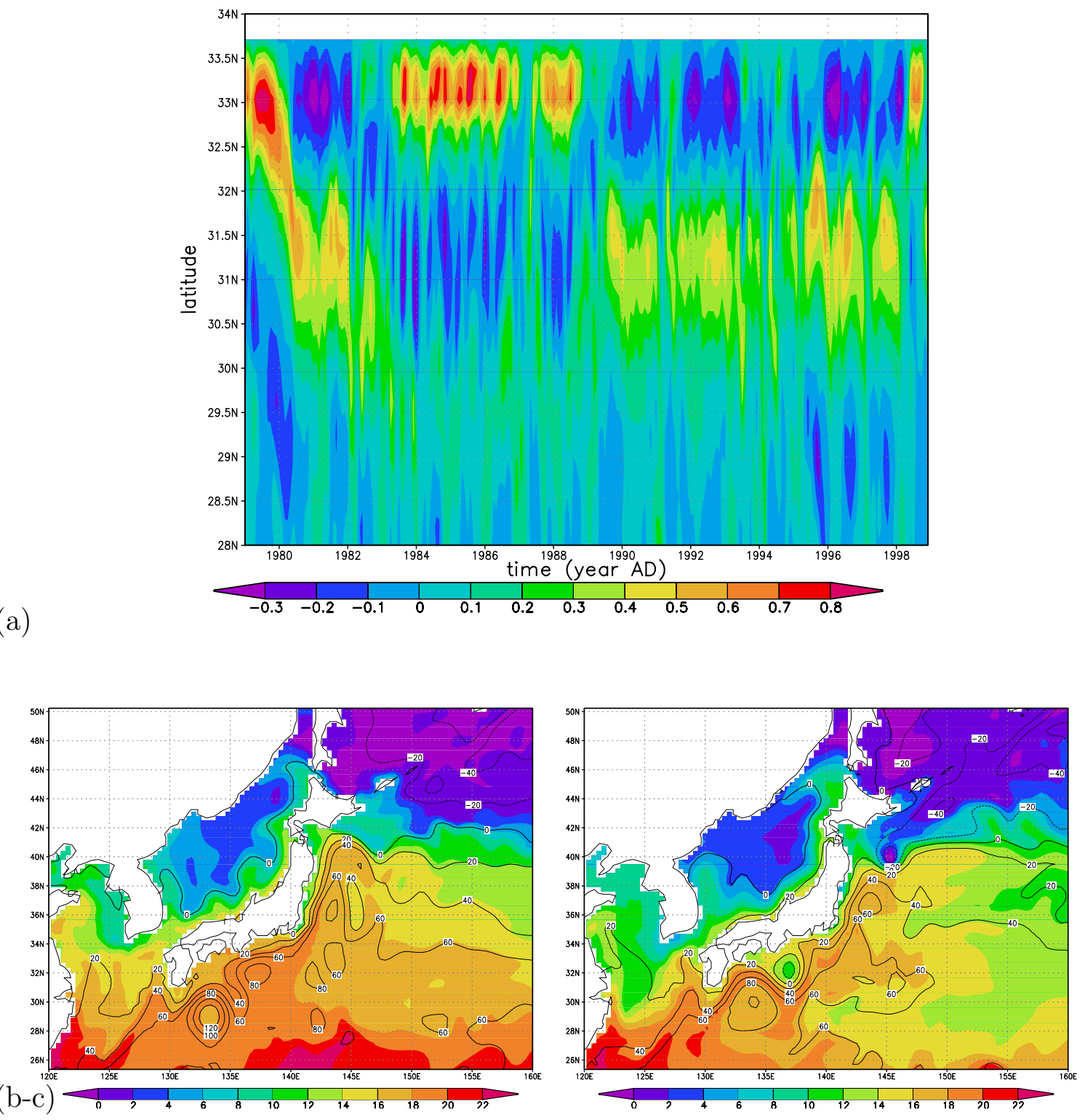

Figure 3: (a) Contour plot of zonal geostrophic velocity $\left(\mathrm{ms}^{-1}\right)$, calculated from meridional SSH gradients in POCM, at $136^{\circ}$ E as a function of latitude for the period 1979-1998. (b-c) Contour plot of monthly mean SSH deviations (cm), superimposed on a shaded contour plot of monthly mean SST ( $\left.{ }^{\circ} C\right)$ for (b) January 1988, representing the small meander state $K_{b}^{P}$ of the Kuroshio in POCM and (c) January 1996, representing the large meander state $K_{c}^{P}$ of the Kuroshio in POCM (cf. Fig. 1). 

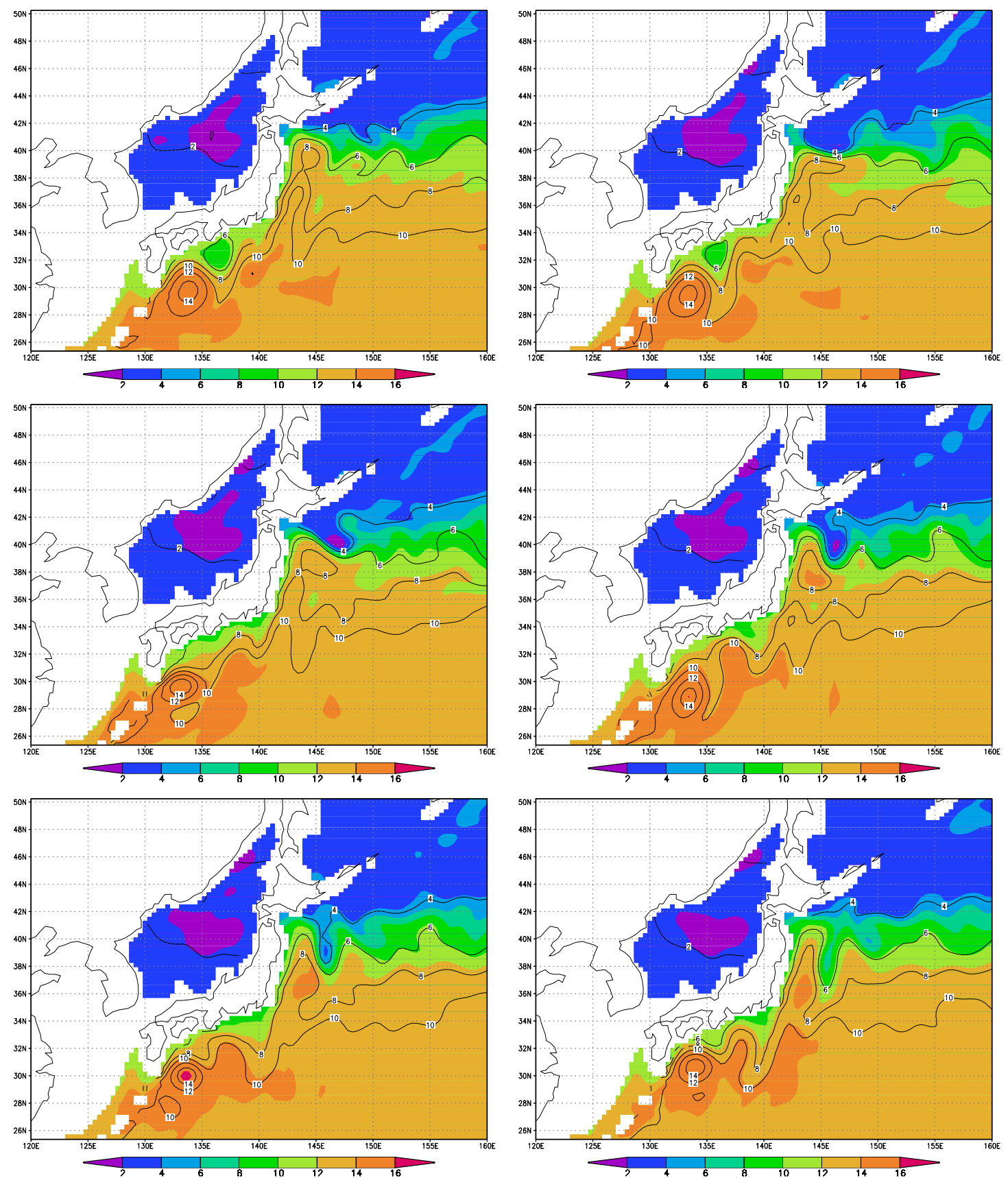

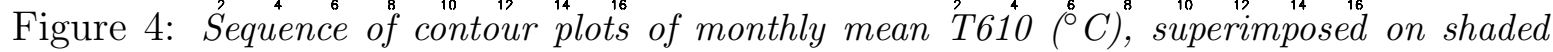
contour plots of monthly mean T310 ( ${ }^{\circ}$ C) from POCM, showing the evolution of the simulated Kuroshio from the large $\left(K_{c}^{P}\right)$ to the small $\left(K_{b}^{P}\right)$ meander state and back during 1998 (see Fig. 3a). The patterns are shown for January and March (upper panels), May and July (middle panels), and September and November (lower panels). 
(a)
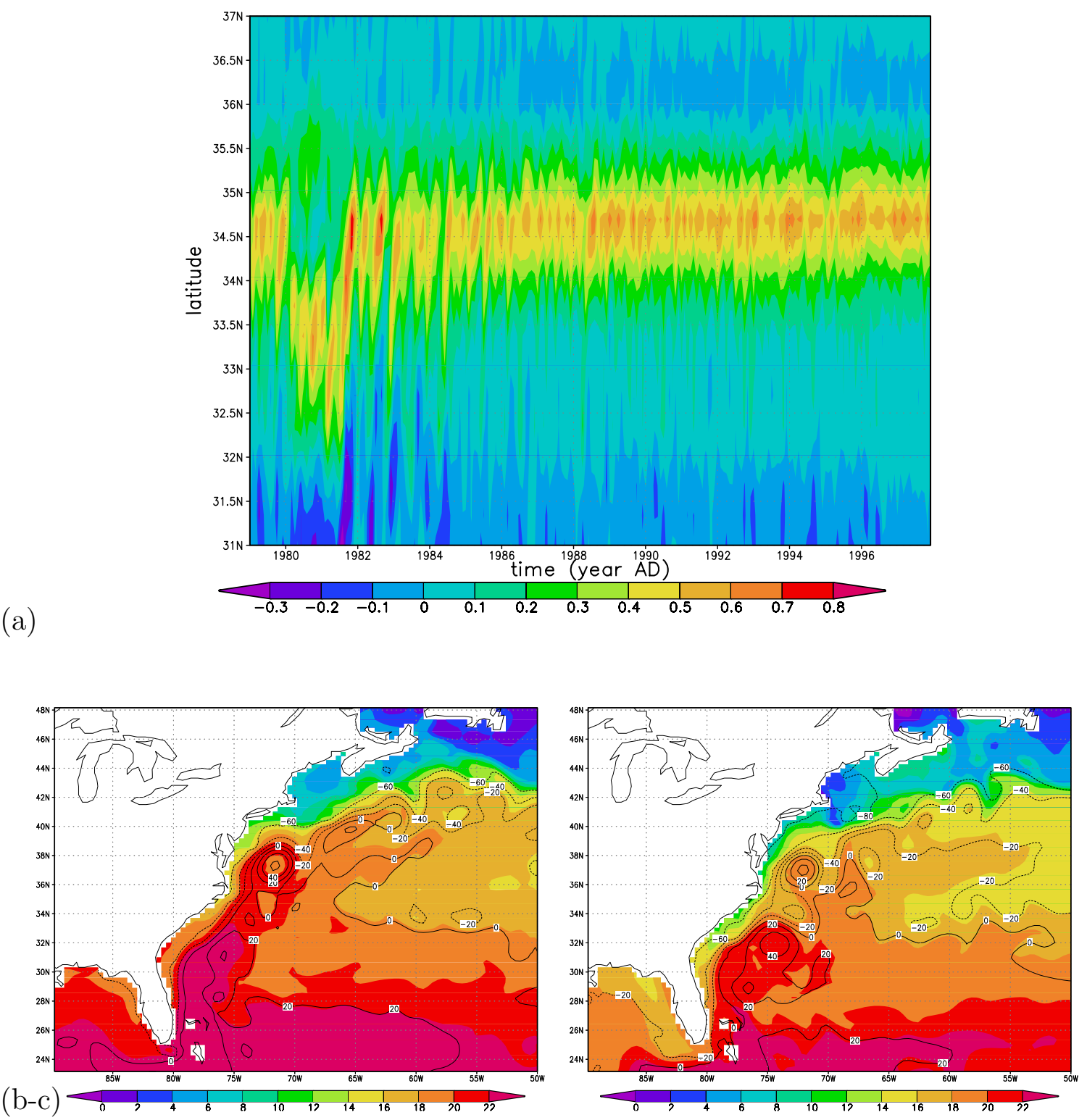

Figure 5: (a) Contour plot of zonal geostrophic velocity $\left(\mathrm{ms}^{-1}\right)$, calculated from meridional SSH gradients in POCM, at $75^{\circ} \mathrm{W}$ as a function of latitude for the period 1979-1997. (bc) Contour plot of monthly mean $S S H$ deviations (cm), superimposed on a shaded contour plot of monthly mean $S S T\left({ }^{\circ} C\right)$ for (b) January 1979, representing Gulf Stream state $G_{b}^{P}$ in POCM and (c) January 1981, representing Gulf Stream state $G_{c}^{P}$ in POCM (cf. Fig. 2). (d-e) Contour plot of monthly mean T610 $\left({ }^{\circ} \mathrm{C}\right)$, superimposed on a shaded contour plot of monthly mean T310 ( ${ }^{\circ}$ C) for (d) January 1979 and (e) January 1981. 

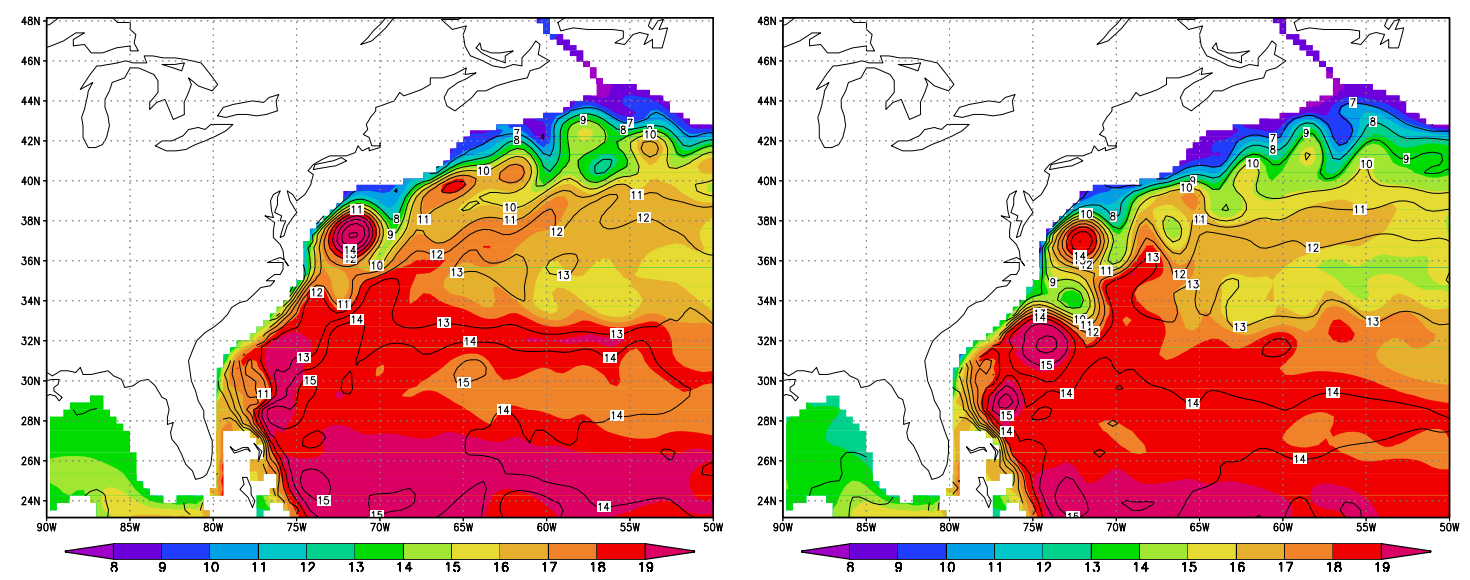

(d-e)

Figure 5 (continued) 
(a)

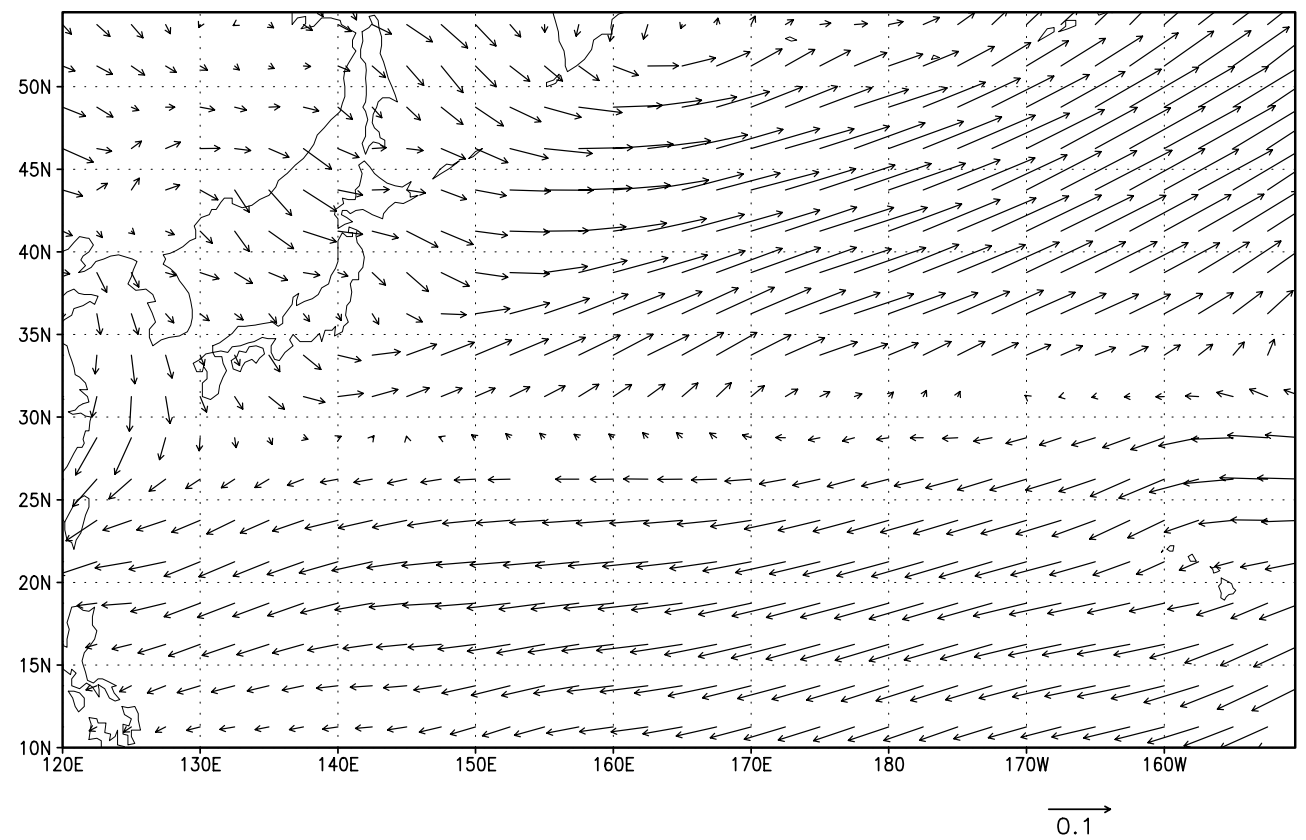

(b)

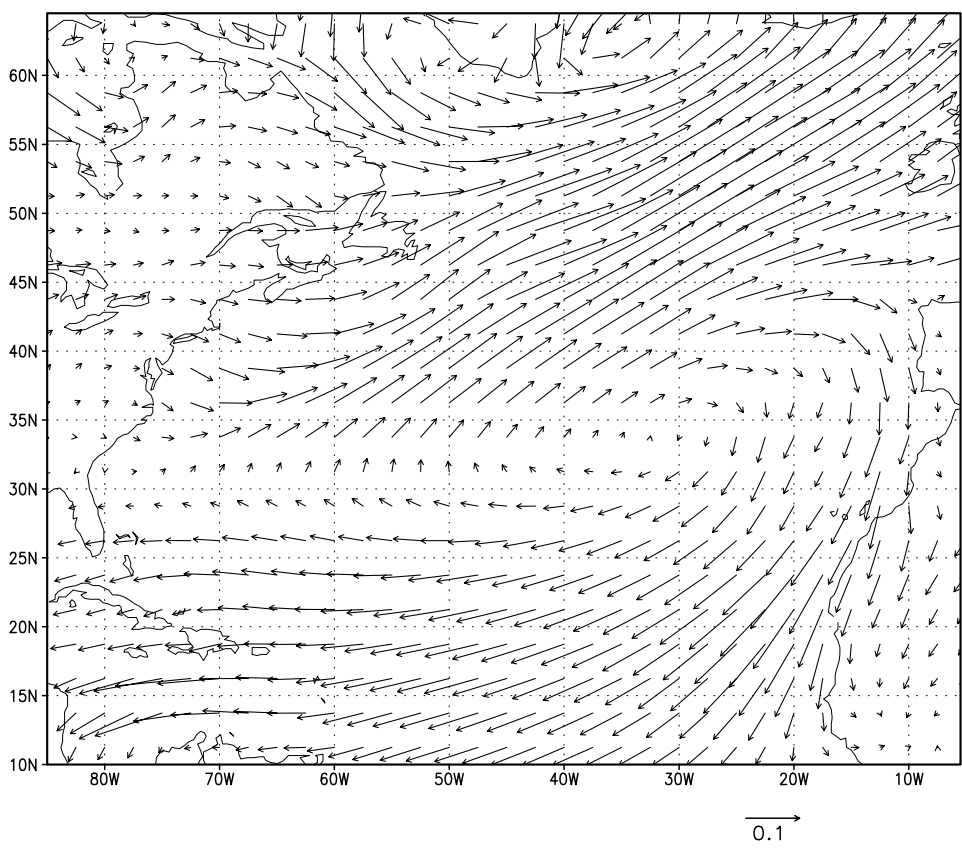

Figure 6: Pattern and magnitude (Pa) of the climatological mean wind stress forcing (Trenberth et al., 1989) on the (a) North Pacific and (b) North Atlantic domain. 


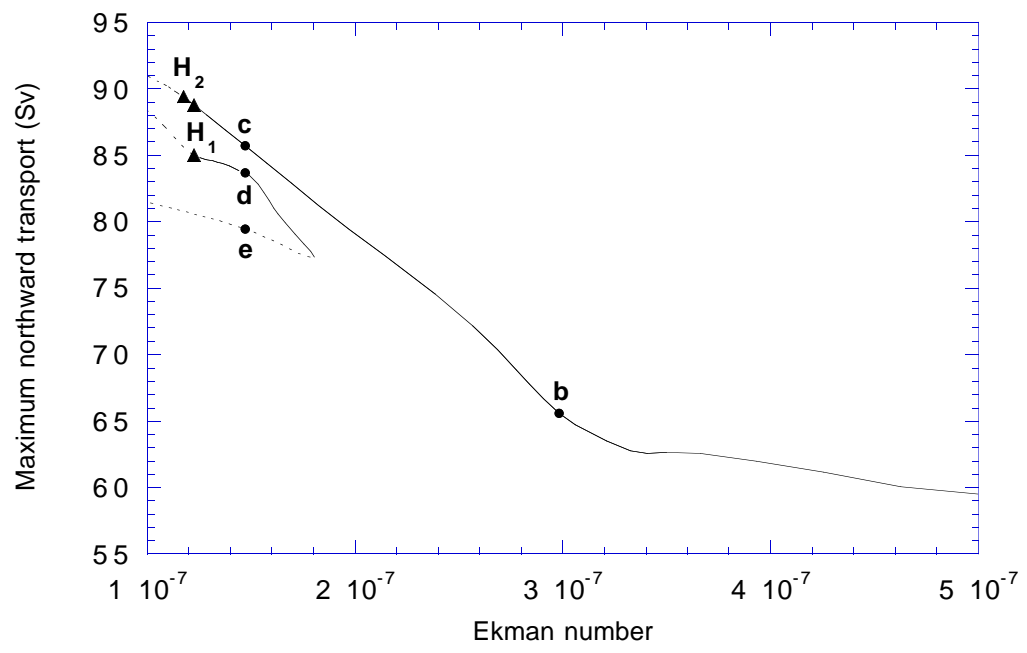

(a)

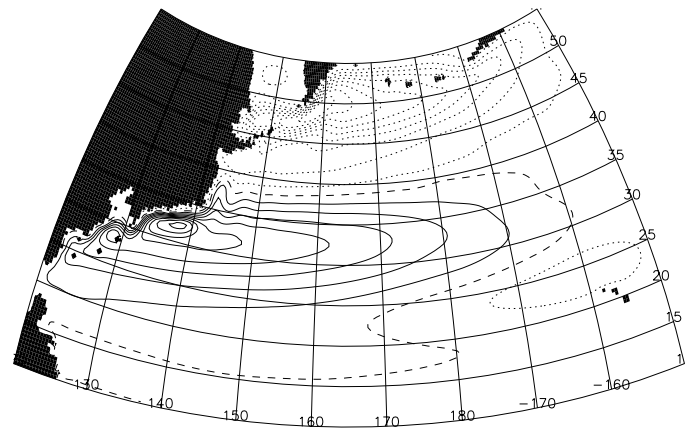

(b)

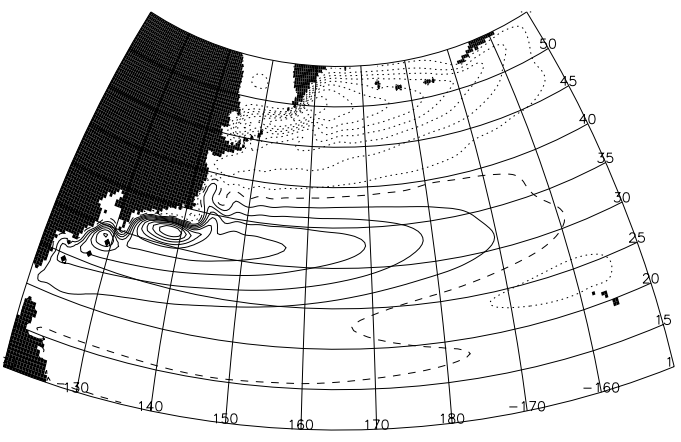

(c)
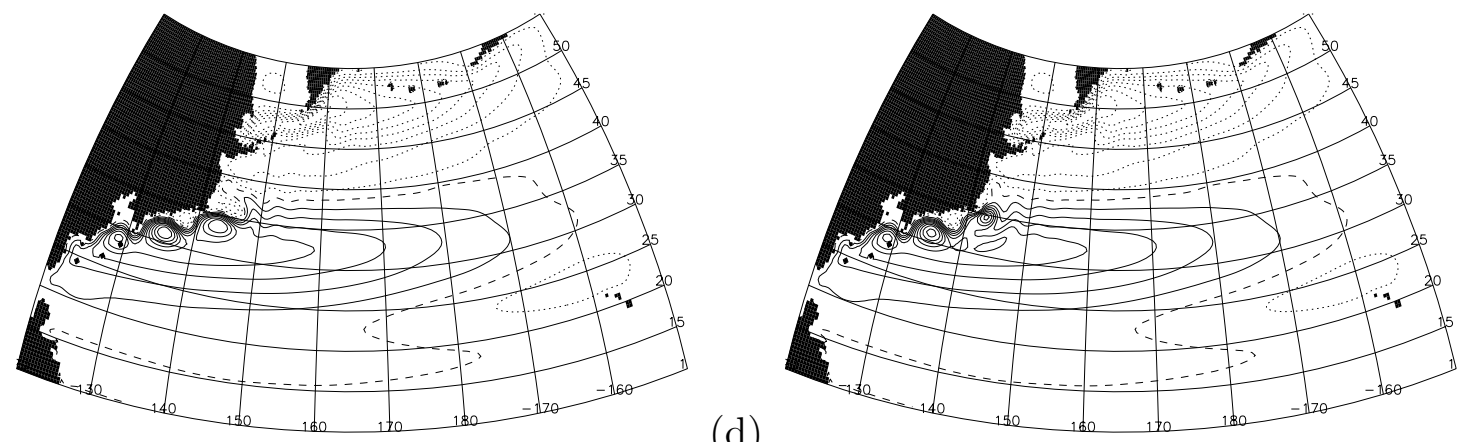

(d)

(e)

Figure 7: (a) Bifurcation diagram for the barotropic shallow water model on the North Pacific domain with the Ekman number as control parameter. Drawn (dotted) branches indicate stable (unstable) steady states, whereas the Hopf bifurcation points are indicated by triangles. The letters b, c, $d$ and e refer to Fig. 7b, 7c, 7d and \%e, respectively. (b) Contour plot of SSH deviations for the steady state solution $K_{b}^{S}$ at point $b$ in (a), that is at $E=3.0 \times 10^{-7}$. The contour levels are scaled with respect to the maximum value of the field. (c) Contour plot of $S S H$ deviations for the steady state solution $K_{c}^{S}$ at point $c$ in (a), that is at $E=1.5 \times 10^{-7}$. (d) Contour plot of SSH deviations for the steady state solution $K_{d}^{S}$ at point $d$ in (a), that is at $E=1.5 \times 10^{-7}$. (e) Contour plot of $S S H$ deviations for the steady state solution $K_{e}^{S}$ at point $e$ in (a), that is at $E=1.5 \times 10^{-7}$. 

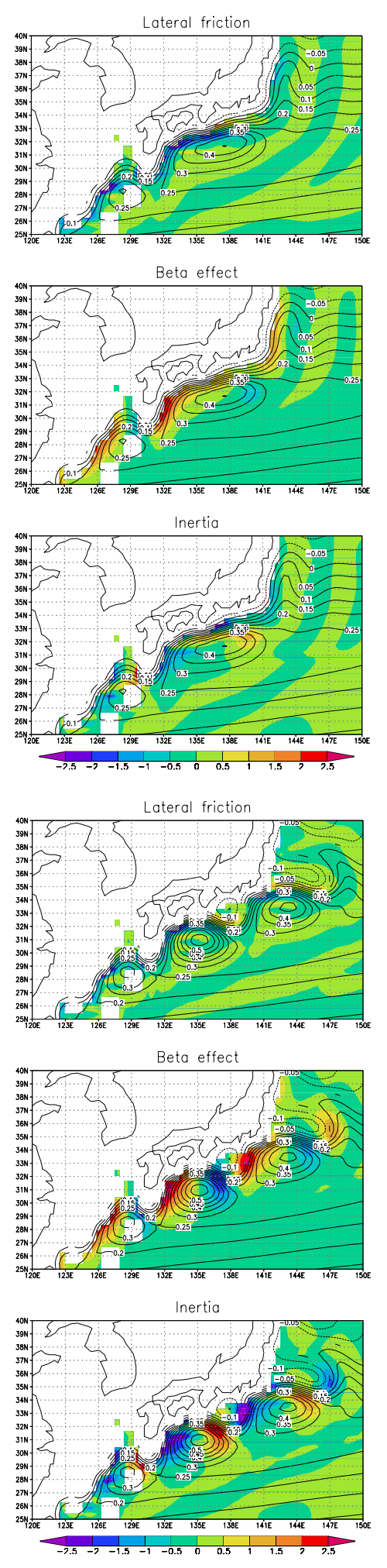

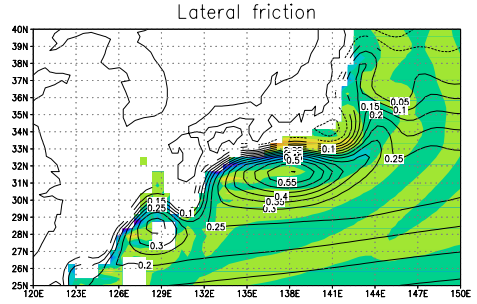

Beta effect

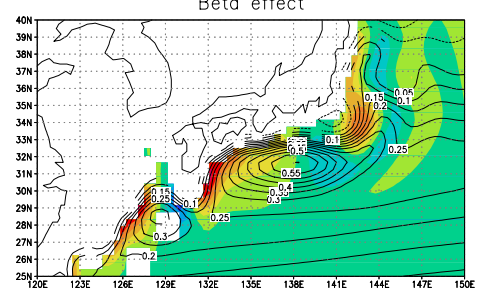

Inertia

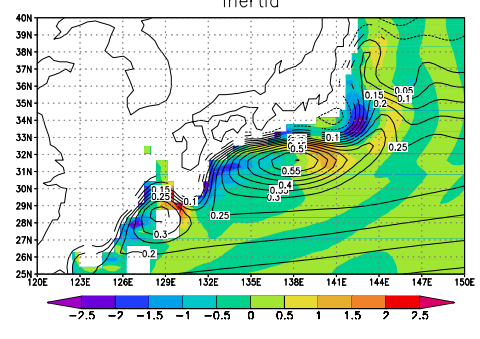

$(\mathrm{a}-\mathrm{b})$

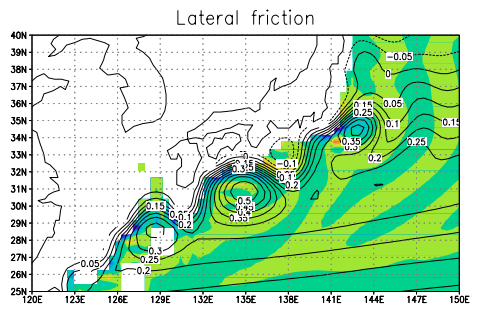

Beta effect

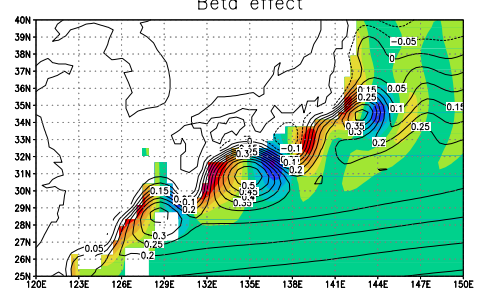

Inertia

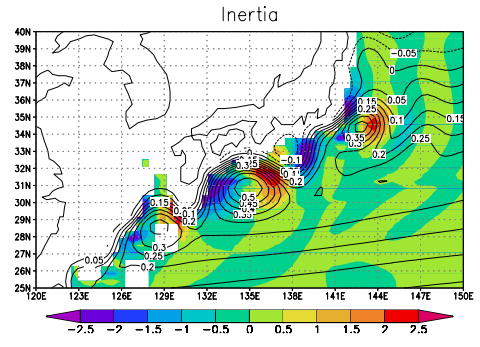

$(\mathrm{c}-\mathrm{d})$

Figure 8: (a) As Fig. Yb, (b) as Fig. Yc, (c) as Fig. 7d, and (d) as Fig. Ye, but for part of the computational domain $[120,150]^{\circ} E \times[25,40]^{\circ} \mathrm{N}$, and superimposed on a shaded contour plot of the value of the lateral friction term $F$ (upper panel), beta effect term $B$ (middle panel) and inertia term I (lower panel) in the barotropic potential vorticity equation (10) (cf. Figs. 1 and 3). 


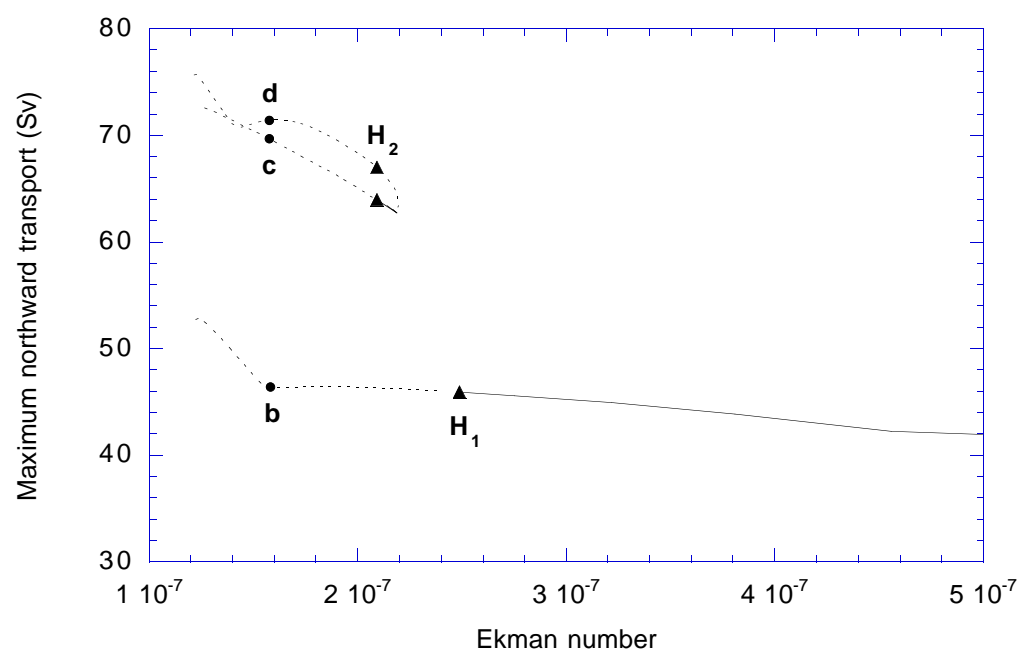

(a)

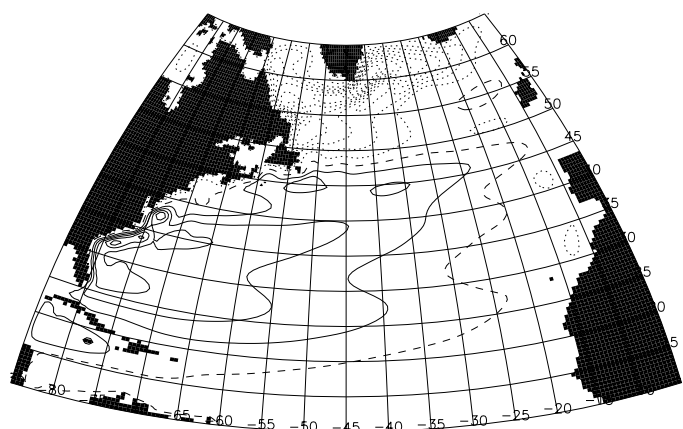

(b)

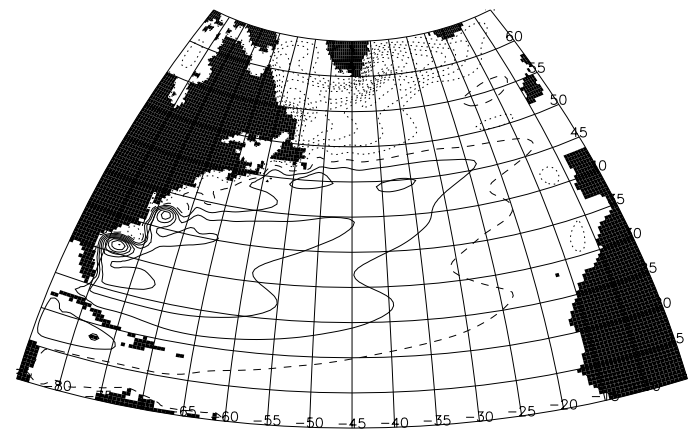

(c)

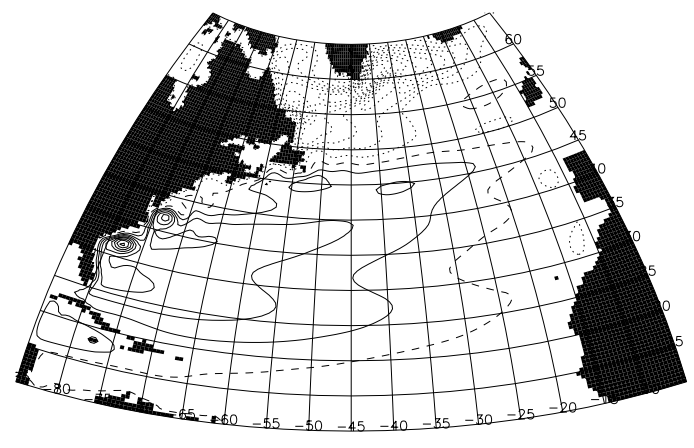

(d)

Figure 9: (a) Bifurcation diagram for the barotropic shallow water model on the North Atlantic domain with the Ekman number as control parameter. Drawn (dotted) branches indicate stable (unstable) steady states, whereas the Hopf bifurcation points are indicated by triangles. The letters $b, c$ and $d$ refer to Fig. $9 b, 9 c$ and $9 d$, respectively. The intersection of the upper branch does not indicate a bifurcation; it is due to the choice of norm. (b) Contour plot of SSH deviations for the steady state solution $G_{b}^{S}$ at point $b$ in $(a)$, that is at $E=1.6 \times 10^{-7}$. The contour levels are scaled with respect to the maximum value of the field. (c) Contour plot of SSH deviations for the steady state solution $G_{c}^{S}$ at point $c$ in $(a)$, that is at $E=1.6 \times 10^{-7}$. (d) Contour plot of SSH deviations for the steady state solution $G_{d}^{S}$ at point $d$ in (a), that is at $E=1.6 \times 10^{-7}$. 

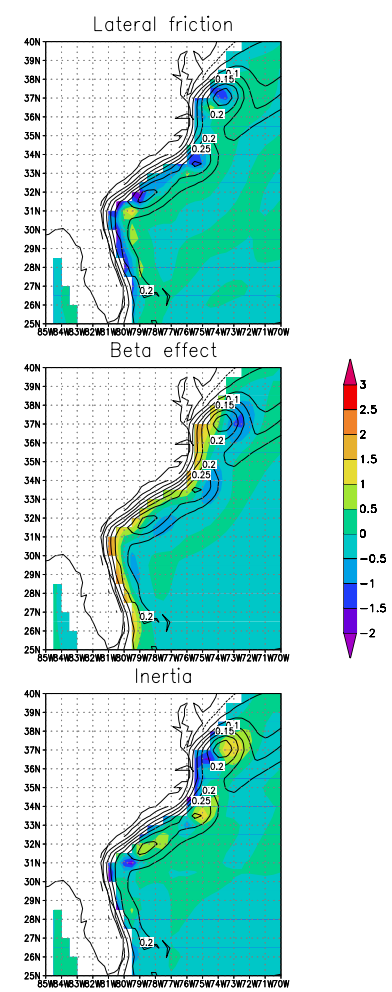

(a)
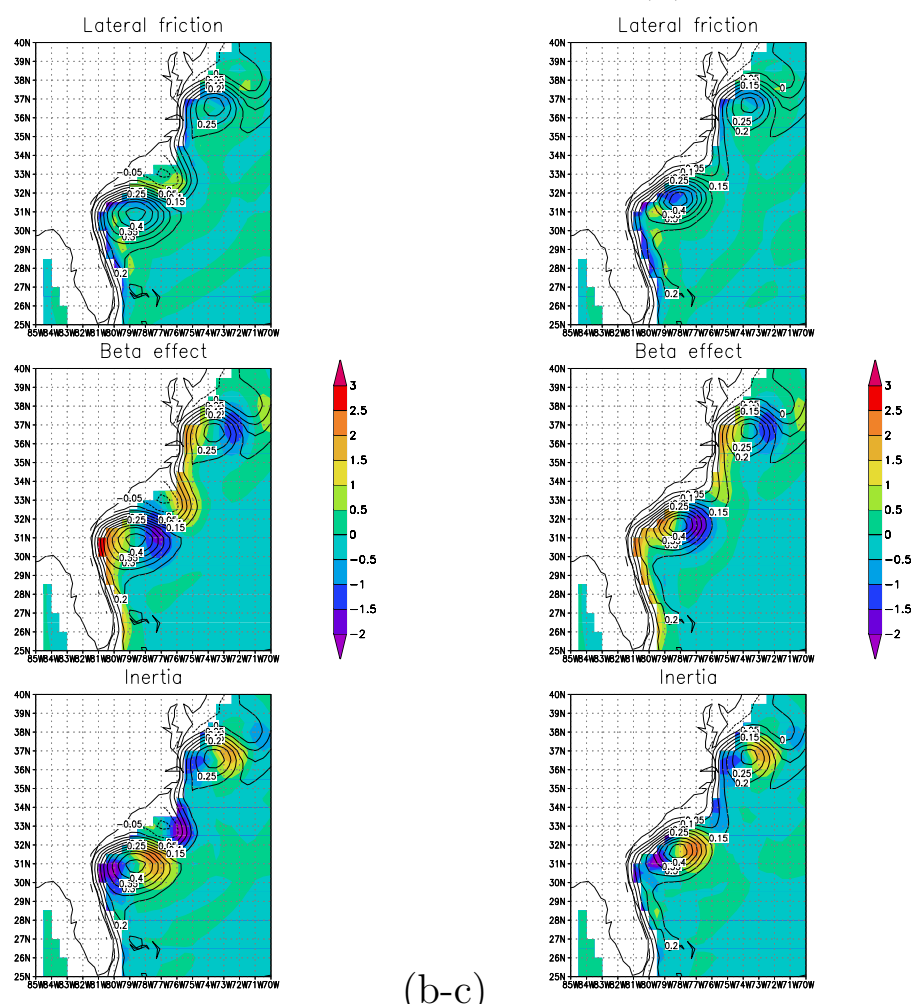

Figure 10: (a) As Fig. 9b, (b) as Fig. 9c, and (c) as Fig. 9d, but for part of the computational domain $[85,70]^{\circ} \mathrm{W} \times[25,40]^{\circ} \mathrm{N}$, and superimposed on a shaded contour plot of the value of the lateral friction term $F$ (upper panel), beta effect term $B$ (middle panel) and inertia term I (lower panel) in the barotropic potential vorticity equation (10) (cf. Figs. 2 and $5)$. 
(a)

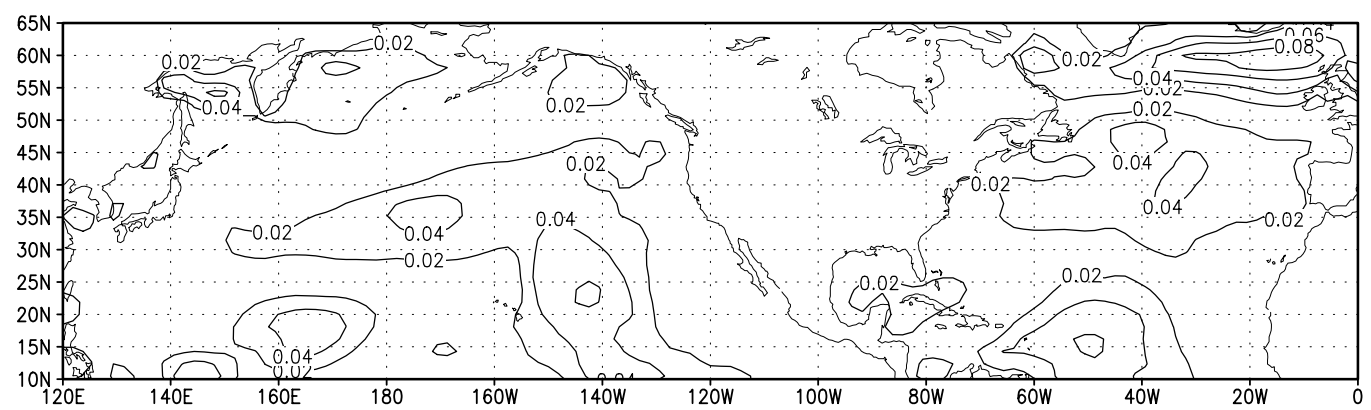

(b)

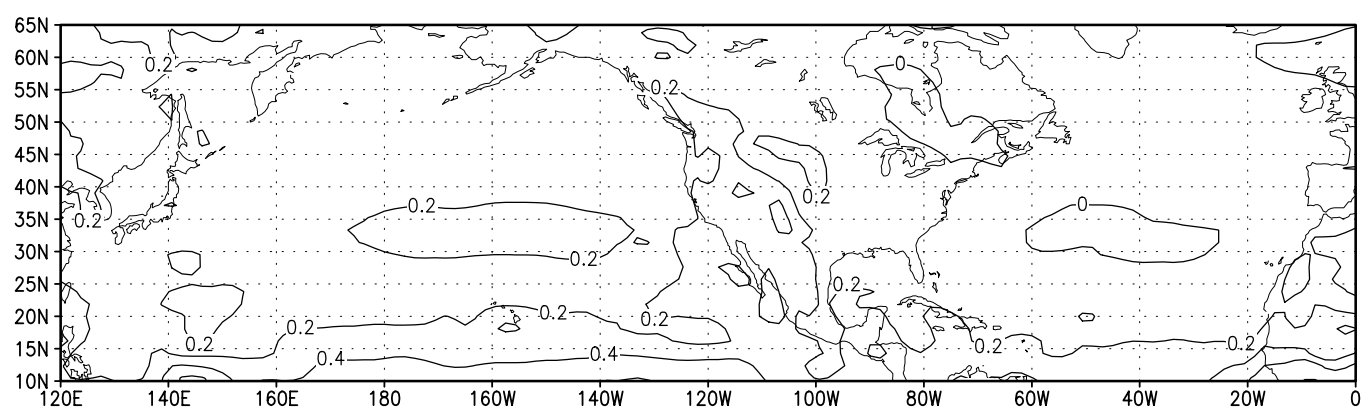

(c)

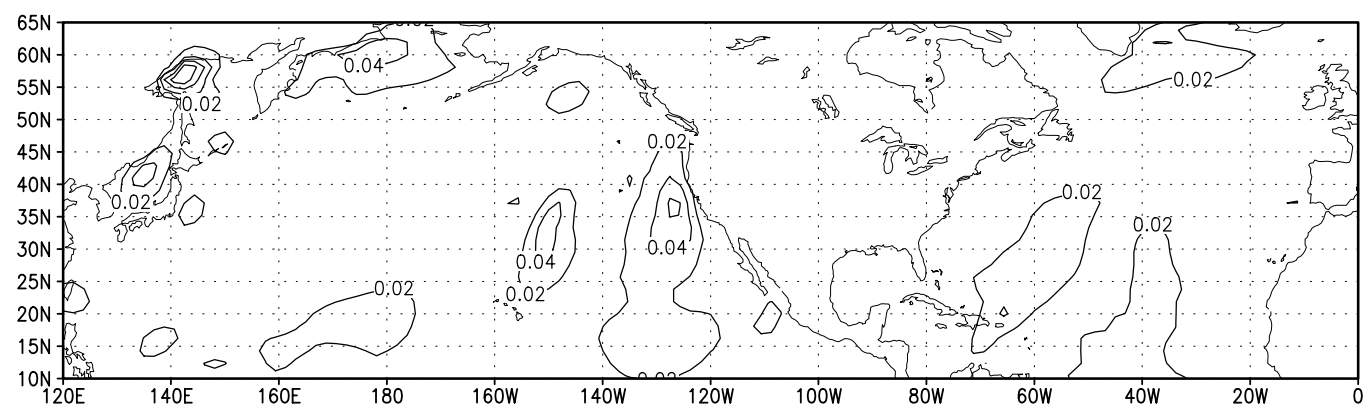

(d)

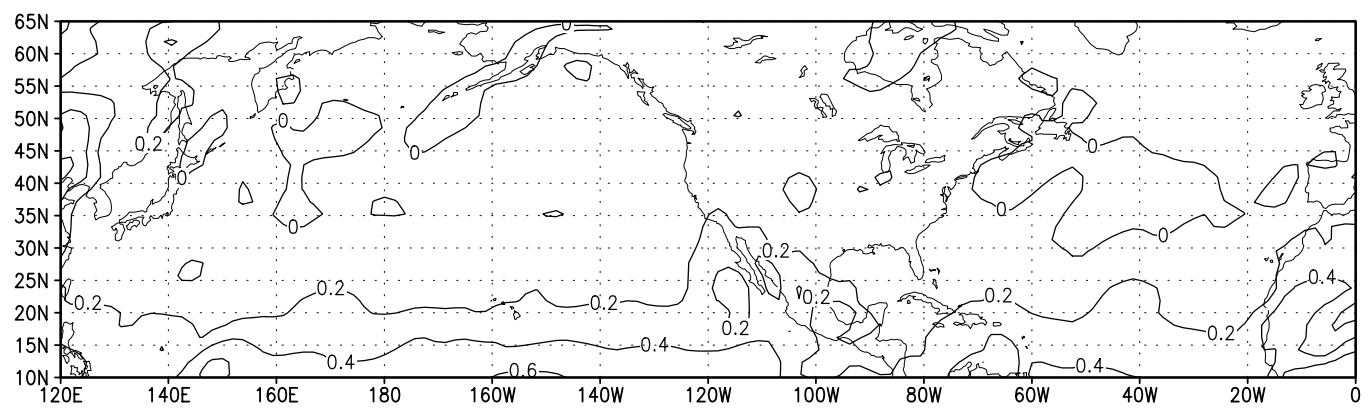

Figure 11: Horizontal map of (a) rms and (b) 1-month lag autocorrelation computed for the zonal wind stress anomaly over the period 1949-1999. (c-d) As in (a-b) except for the meridional component of the wind stress. 


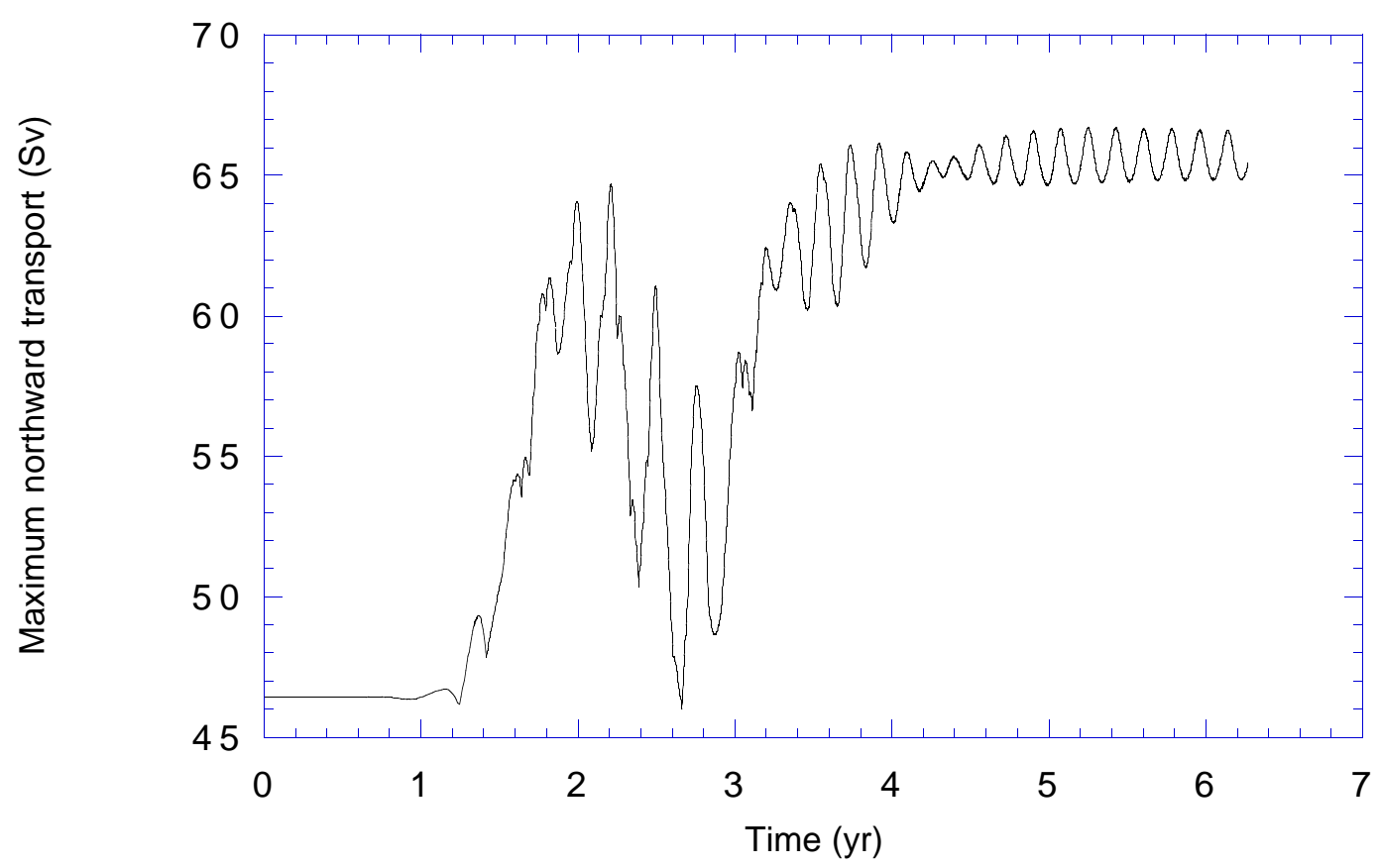

(a)

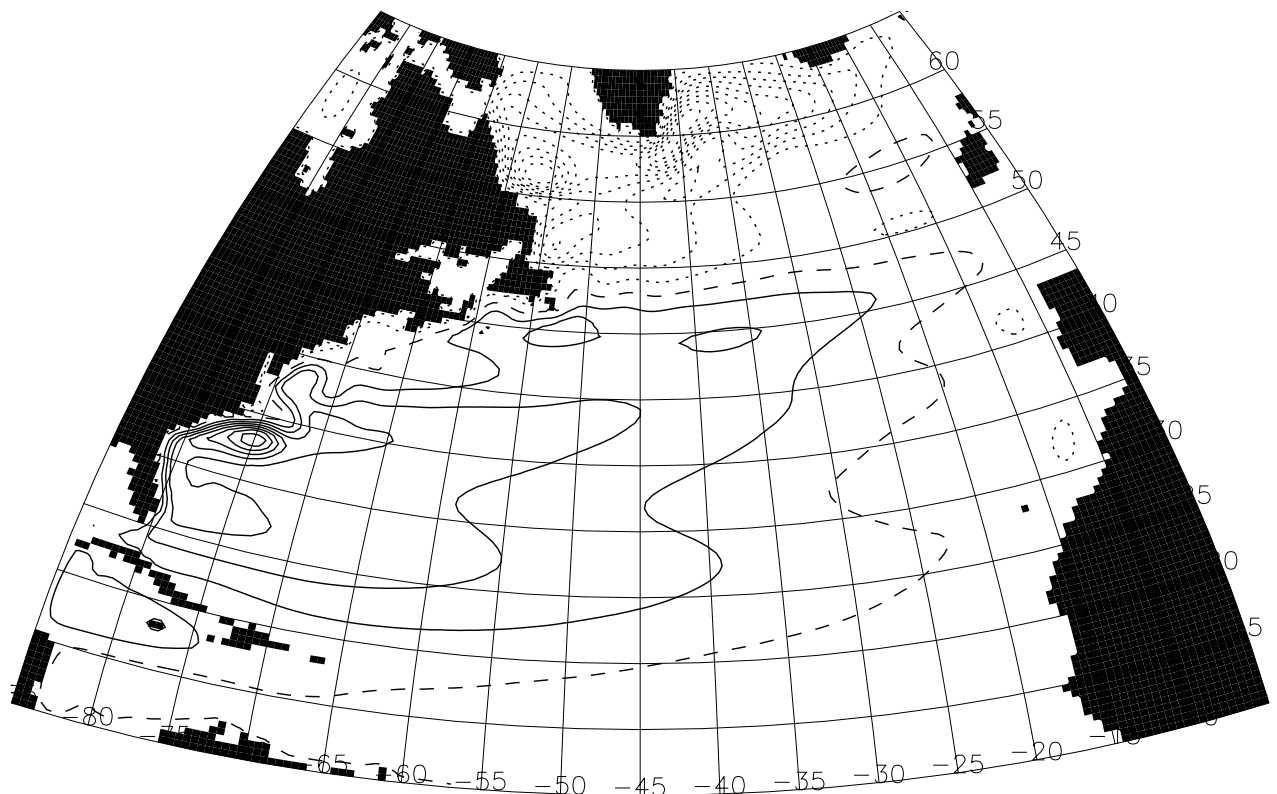

(b)

Figure 12: (a) Maximum northward transport for the 6-year time integration of the barotropic shallow water model on the North Atlantic domain with the climatological mean wind stress of Fig. $6 b$ as forcing and $E=1.6 \times 10^{-7}$. (b) Mean $S S H$ field of the stable limit cycle (cf. Fig. 5c). 


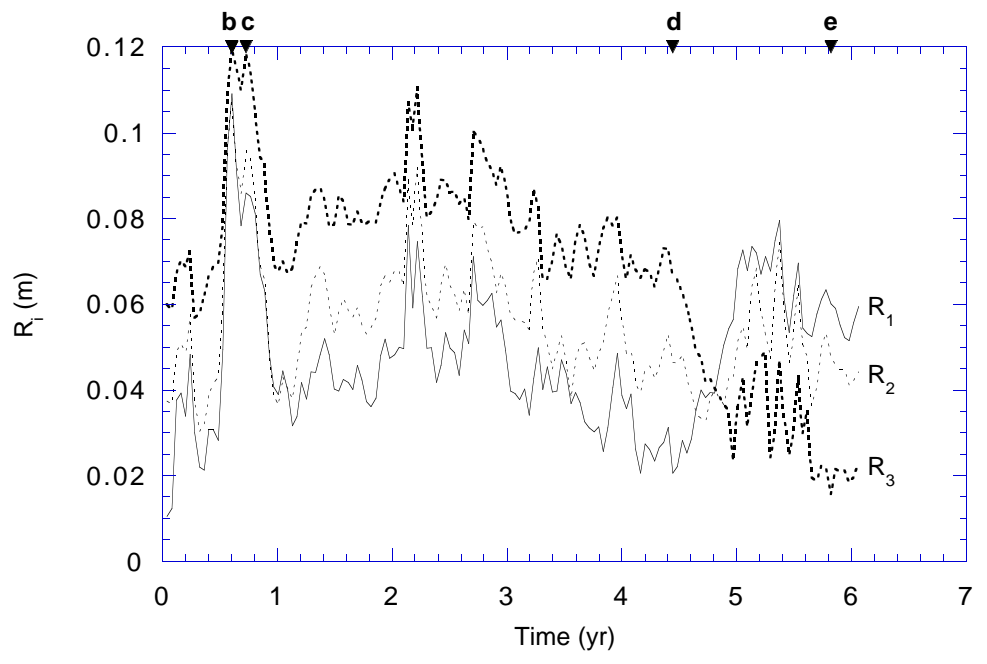

(a)

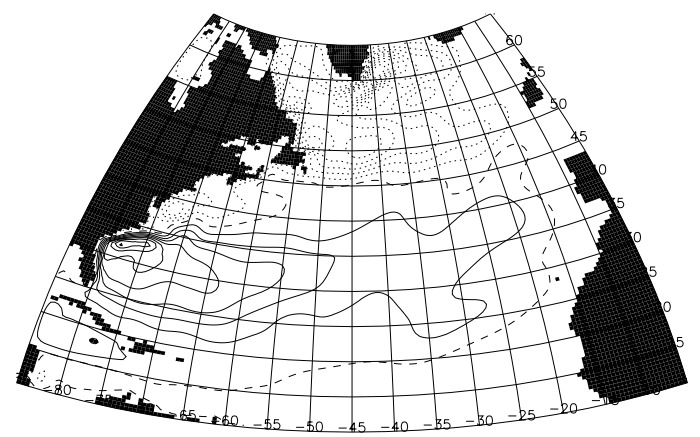

(b)
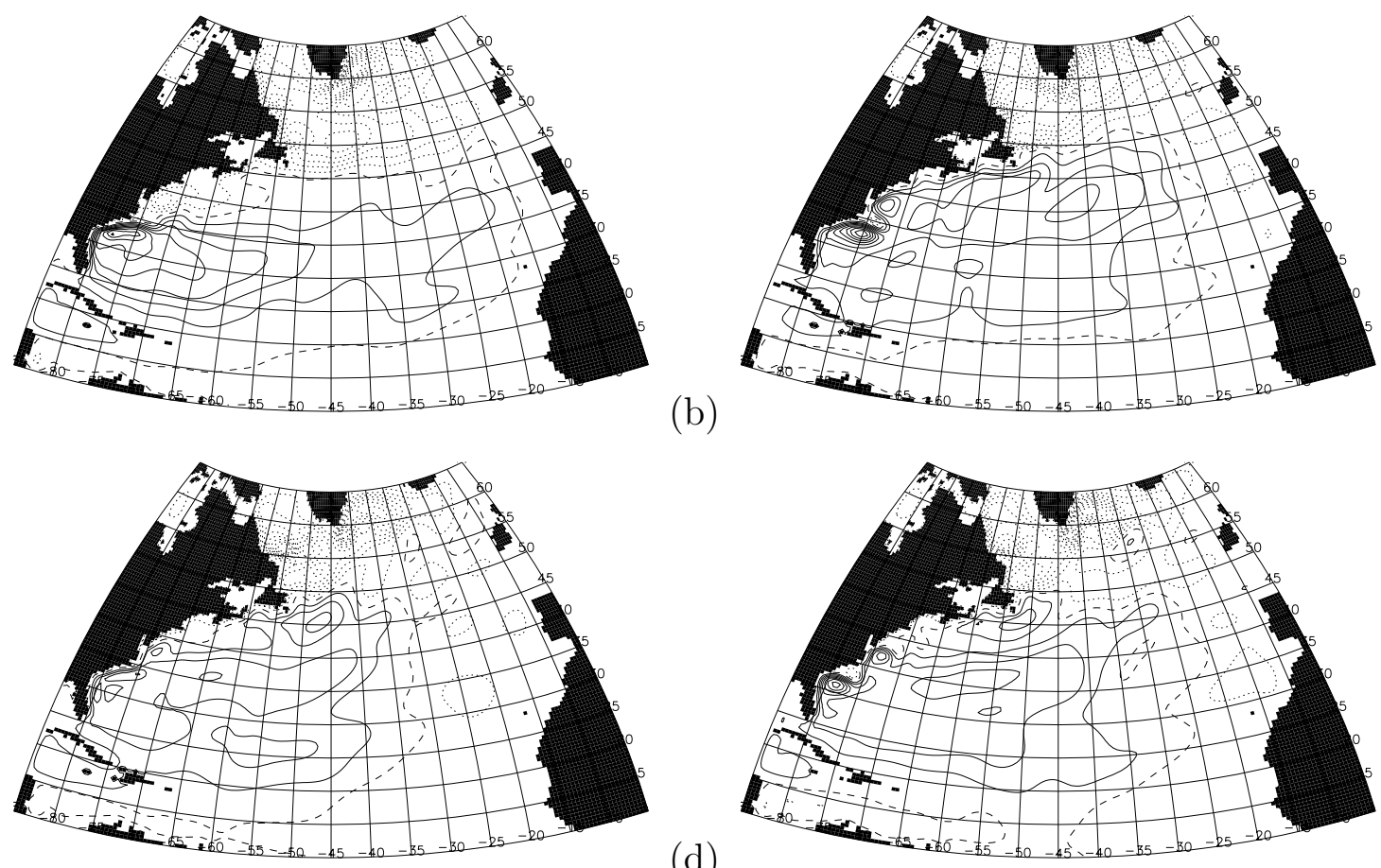

(c)

(d)

(e)

Figure 13: Output of the 6-year time integration of the barotropic shallow water model on the North Atlantic domain with the stochastic forcing added to the climatological mean forcing and $E=1.6 \times 10^{-7}$. (a) $R_{i}$ values, defined in (11), as a function of time; $i=1$ (drawn) for the steady state $G_{b}^{S}$ (Fig. 10a), $i=2$ (thin dotted) for the steady state $G_{d}^{S}$ (Fig. 10c), and $i=3$ (thick dotted) for the steady state $G_{c}^{S}$ (Fig. 10b). The letters b-e refer to Figs. 13b-e. (b-e) Snapshots of SSH deviations for (b) $t=0.61 \mathrm{yr}$, (c) $t=0.73 \mathrm{yr}$ (cf. Fig. 12b), (d) $t=4.44$ yr (cf. Fig. 9b) and (e) $t=5.82$ yr (cf. Fig. 9c). 


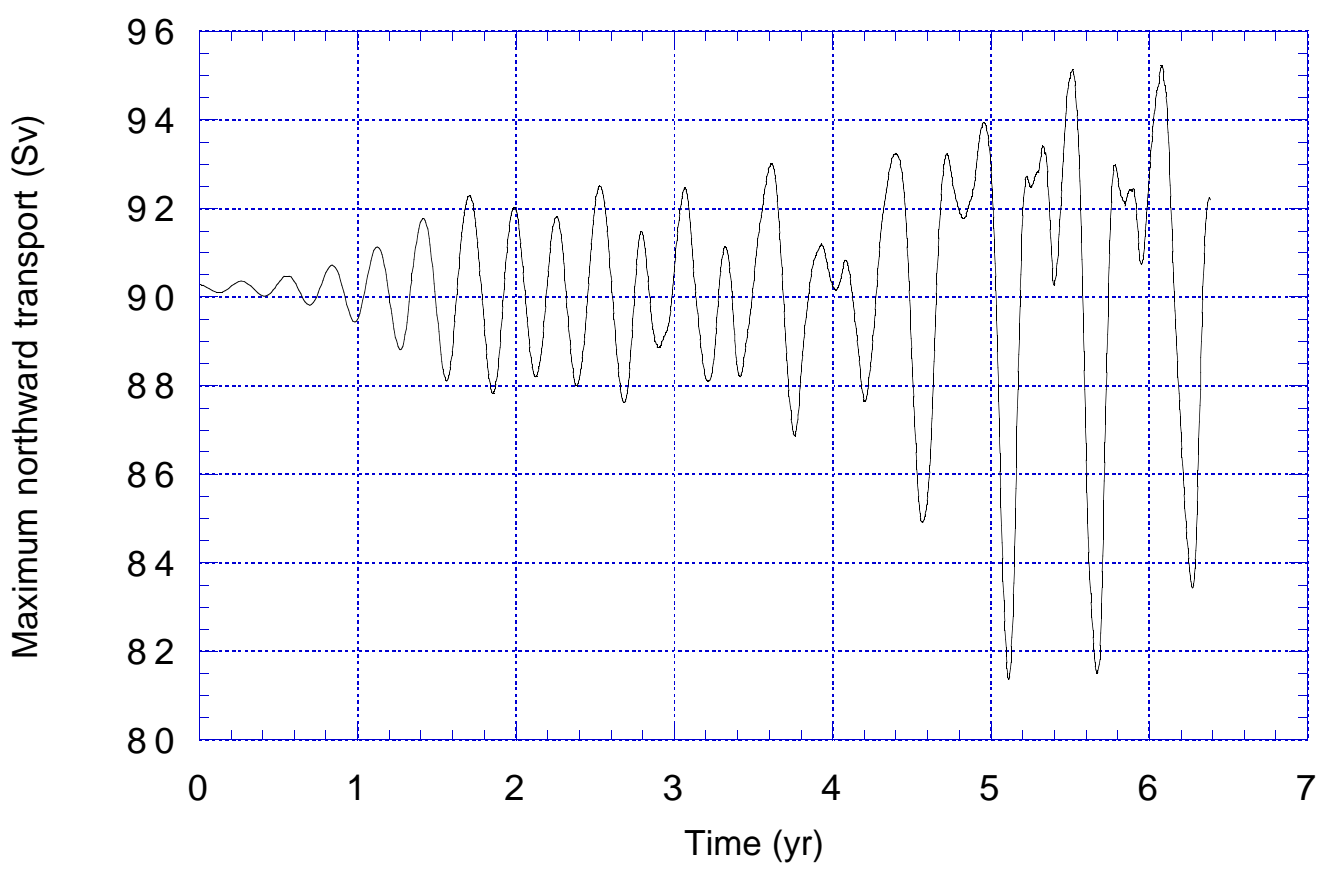

(a)

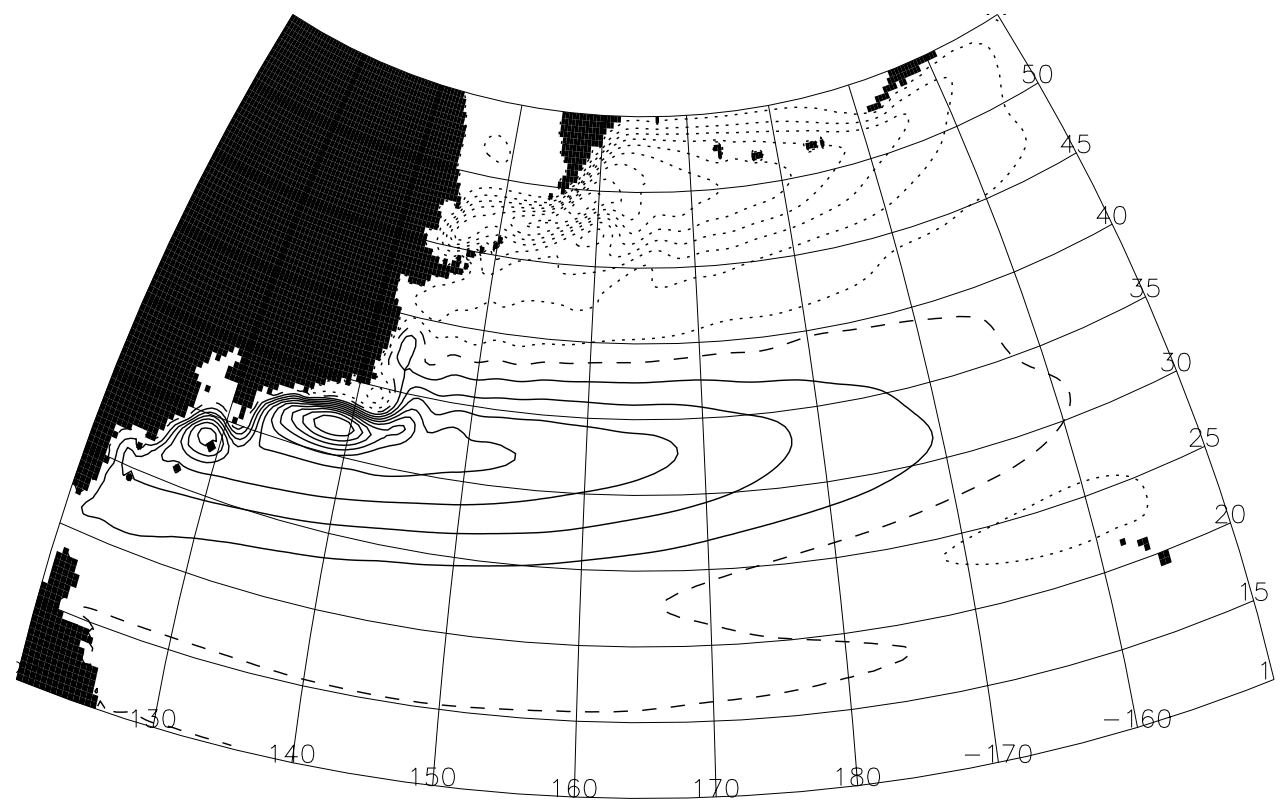

(b)

Figure 14: (a) Maximum northward transport for the 6-year time integration of the barotropic shallow water model on the North Pacific domain with the climatological mean wind stress of Fig. 6 a as forcing and $E=1.1 \times 10^{-7}$. (b) Mean SSH field over the last year of the integration (cf. Fig. 7c). 


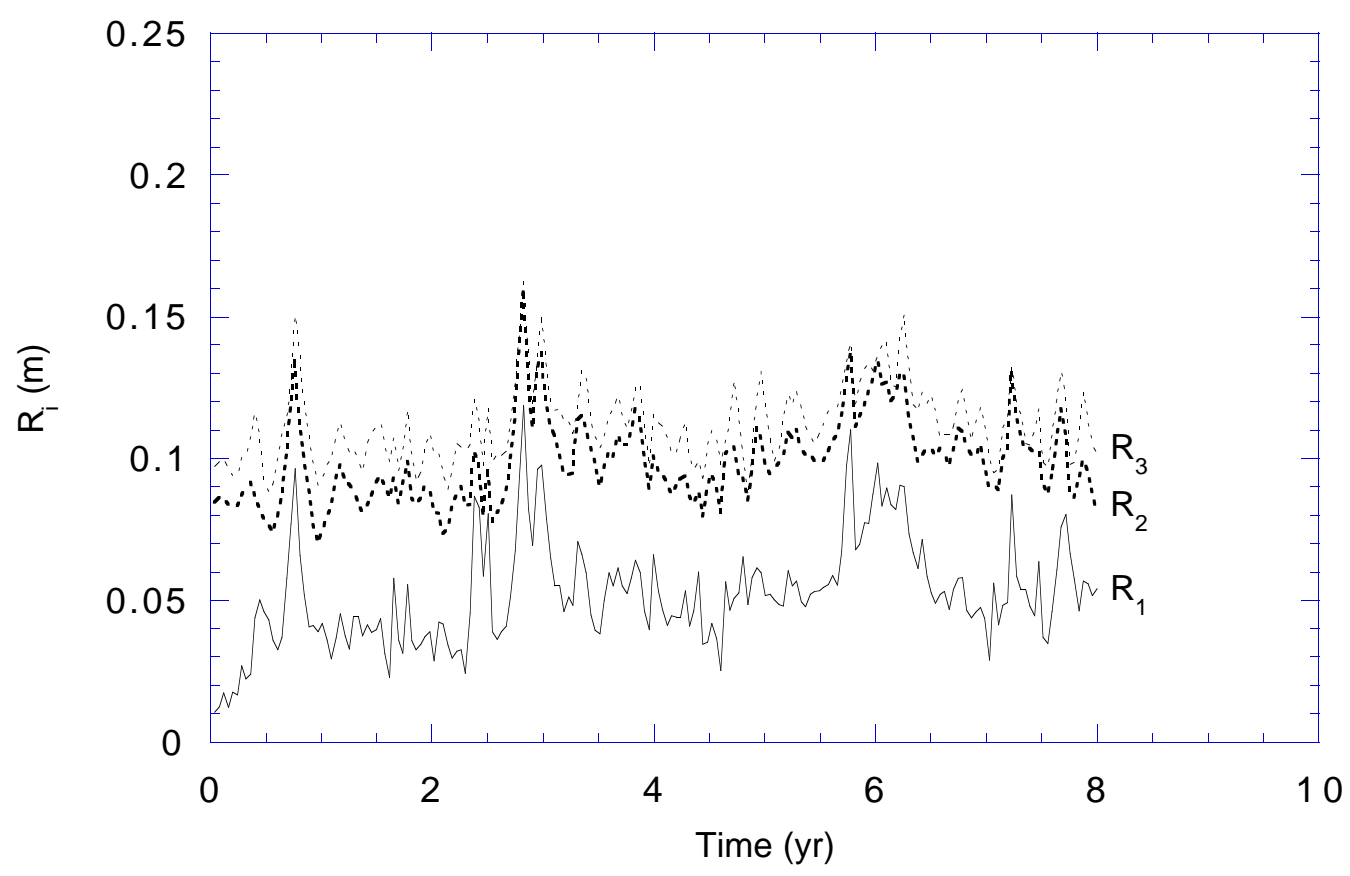

(a)

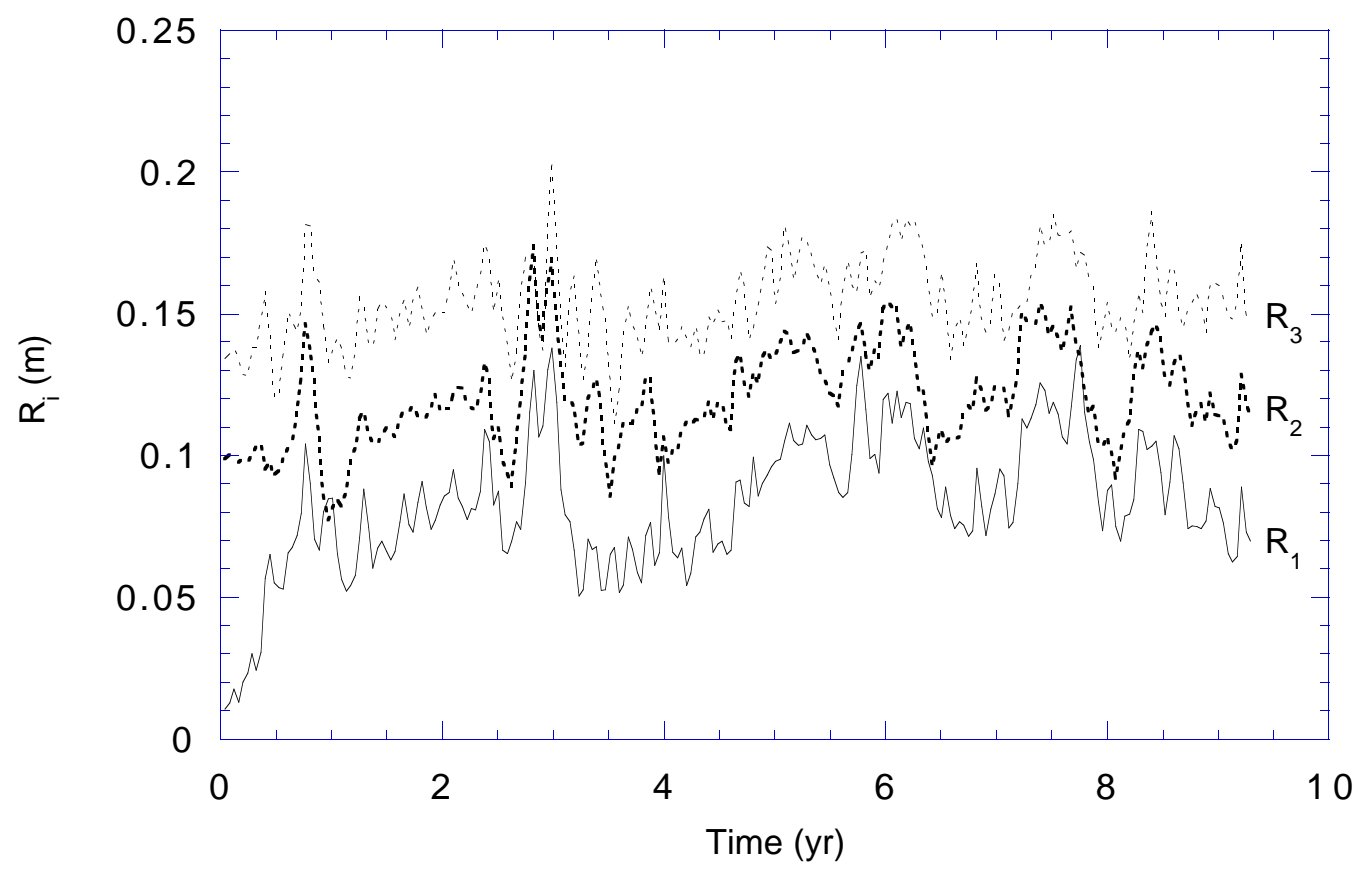

(b)

Figure 15: (a-b) $R_{i}$ values, defined in (11), as a function of time, for the time integrations of the barotropic shallow water model on the North Pacific domain with the stochastic forcing added to the climatological mean forcing and (a) $E=1.1 \times 10^{-7}$ and (b) $E=7.3 \times 10^{-8}$; $i=1$ (drawn) for the respective steady state similar to $K_{c}^{S}$ (Fig. 8b), $i=2$ (thick dotted) for the respective steady state similar to $K_{e}^{S}$ (Fig. 8d), and $i=3$ (thin dotted) for the respective steady state similar to $K_{d}^{S}$ (Fig. 8c). 